\title{
1 AdRoit: an accurate and robust method to infer complex 2 transcriptome composition
}

3

\section{Affiliations}

\section{Abstract}

Tao Yang ${ }^{1}$, Nicole Alessandri-Haber ${ }^{1}$, Wen Fury ${ }^{1}$, Michael Schaner ${ }^{1}$, Robert Breese ${ }^{1}$, Michael LaCroix-Fralish², Jinrang Kim ${ }^{1}$, Christina Adler ${ }^{1}$, Lynn E. Macdonald ${ }^{1}$, Gurinder S. Atwal ${ }^{1}$, Yu Bai $^{1,}{ }^{*}$

1. Regeneron Pharmaceuticals, Inc., Tarrytown NY 10591

2. Cellular Longevity, Inc., San Francisco, CA 94103

${ }^{*}$ Corresponding author

Bulk RNA sequencing technology provides the opportunity to understand biology at the whole transcriptome level without the prohibitive cost of single cell profiling. Advances in spatial transcriptomics enable to dissect tissue organization and function by genome-wide gene expressions. However, the readout of both technologies is the overall gene expression across potentially many cell types without directly providing the information of cell type constitution. Although several in-silico approaches have been proposed to deconvolute RNA-Seq data composed of multiple cell types, many suffer a deterioration of performance in complex tissues. Here we present AdRoit, an accurate and robust method infer the cell composition from transcriptome data comprised of multiple cell types. AdRoit uses gene expression profile obtained from single cell RNA sequencing as a reference. It employs an adaptive learning approach to correct the sequencing technique difference between the single cell data and the bulk or spatial transcriptome data, enabling cross-platform readout comparability. Our 
26 systematic benchmarking and applications, which include deconvoluting complex mixtures that

27 encompass 30 cell types, demonstrate its superior sensitivity and specificity compared to other

28 existing methods as well as its utilities. In addition, AdRoit is computationally efficient and runs

29 orders of magnitude faster than many existing methods.

30

\section{Introduction}

32 RNA sequencing is a powerful tool to understand the biology of normal and disease tissues at

33 the whole transcriptome level. It helps to elucidate disease mechanism and identify potential

34 treatment targets ${ }^{1}$. Due to the presence of heterogeneous cell populations, the bulk tissue

35 transcriptome only characterizes the overall gene expression across multiple cell types. The cell

36 type identifies and their prevalence remain unknown in the bulk data. However, knowledge of

37 the cell type composition is often critical to understand the biology. For instance, the

38 constitution of stromal and immune cells sculpts the tumor microenvironment, which is

39 essential in cancer progression or response to immune check point inhibition ${ }^{2-6}$. Excessive

40 expression of cytokines in particular leukocyte types underlines the etiology of many chronic

41 inflammatory diseases ${ }^{7-11}$. Such information cannot be directly read out from the bulk RNA-Seq.

42

43 Recent breakthroughs in spatial transcriptomics methods enable characterizing whole

44 transcriptome-wise gene expressions at spatially resolved locations in a tissue section ${ }^{12}$.

45 However, it remains challenging to reach a single cell resolution while measuring tens of

46 thousands of genes transcriptome-wise. Some widely used technologies can achieve a

47 resolution of $50-100 \mu \mathrm{m}$, equivalent to $3-30$ cells depending on the tissue type ${ }^{12,13}$. The 
48 transcripts therein may originate from more than one cell type, resulting in another form of

49 RNA-Seq data of multiple cell types. Unlike the bulk RNA-Seq, the profiling data at each spot contains substantial dropouts as merely a few cells are sequenced, imposing additional

51 challenges to demystify the cell type content. We refer to bulk RNA-Seq data and spatial

52 transcriptome data at the multi-cell resolution as compound RNA-Seq data hereafter.

54 The rapid development of single-cell RNA-Seq (scRNA-Seq) technologies has allowed for cell-

55 type specific transcriptome profiling ${ }^{14}$. It provides the information missing from the RNA-Seq

56 data of tissues comprised of multiple cell types (e.g., bulk RNA-Seq). Nevertheless, the

57 technologies have low sensitivity and substantial noise due to the high dropout rate and the

58 cell-to-cell variability. Consequently, scRNA-Seq technologies require a large number of cells

59 (thousands to tens of thousands) to ensure statistical significance in the results. In addition, the cells must remain viable during the capture. These requirements render the scRNA-Seq

61 technologies costly, prohibiting their application in clinical studies that involve many subjects or

62 cannot allow real time tissue dissociation and cell capture. Furthermore, scRNA-Seq

63 technologies may not be well suited to characterizing cell-type proportions in solid tissues

64 because the dissociation and capture steps may not have the same efficiency for different cell 65 types ${ }^{15-17}$.

67 Because sequencing at the single cell level is not always feasible and also for the purpose of

68 better interpreting many highly valuable existing bulk RNA-Seq or spatial transcriptomic data 69 sets, in silico approaches have been developed to infer cell type proportions from compound 
RNA-Seq data ${ }^{18-24}$. The most common strategy is to conduct a statistical inference through the maximum likelihood estimation $(\mathrm{MLE})^{25}$ or the maximum a posterior probability estimation $(\mathrm{MAP})^{26}$ on a constrained linear regression framework, wherein the unobserved mixing proportion of a finite number of cell types are part of the latent variables to be optimized ${ }^{19,21-}$ ${ }^{24}$. The deconvolution methods are often applied to dissect the immune cell compositions in blood samples ${ }^{27-31}$. However, their performance in more complex tissues, such as the nervous, ocular, respiratory or gastrointestinal organs, remains unclear. These tissues often contain many cell types $\left(10-10^{2}\right)$ and the difference among related cells can be subtle, rendering the deconvolution a challenging task. For example, a recent study on the mouse nervous system using scRNA-Seq found more than 200 cell clusters and many are highly similar neuronal subtypes ${ }^{32}$.

Earlier works often utilized the transcriptome profiling of the purified cell populations to estimate the gene expressions of cell type (e.g. Cibersort) ${ }^{19}$. More recently, acquiring cell type specific expression from the scRNA-Seq data was shown to be an intriguing alternative ${ }^{21-24}$. Although it provides higher throughput by measuring multiple cell types in one experiment, profiling at the single cell level has a high noise floor. The accuracy of deconvolution using scRNA-Seq data as the reference may be affected by the data noise if not treated properly. Moreover, the platform difference between the compound data and the single cell data cannot be ignored. 
91 To overcome these challenges, additional information from the data may be considered. A

92 recent method that weighs genes according to their expression variances across samples

93 greatly improved the accuracy ${ }^{22}$, highlighting the importance of gene variability in inferring cell

94 type composition. Some other methods and applications have pointed out the importance of

95 cell type specific genes $24,28,31,33$. In these works, the cell type specific expression was only used

96 to select the input genes (e.g., markers). Nonetheless, it measures how informative a gene is in

97 distinguishing cell types and thus can be incorporated as a part of the model. To address the

98 platform difference between the compound data and the single cell data it is usually assumed

99 there exists a single scaling factor or a linearly scaled bias for all genes that can be learned and

100 corrected accordingly ${ }^{22,23}$. This assumption does not hold, as the impact of changing platforms

101 is different for each gene. Though learning a uniform scaling factor would correct the difference

102 in most genes, a few genes that remain significantly biased can easily confound the estimation,

103 especially under a linear model framework. Thus, a gene-wise correction should be considered.

104

105 In this work, we present a new deconvolution method, AdRoit. A unified framework that jointly

106 models the gene-wise technology bias, as well as the cell type specificity and cross-sample

107 variability of genes. The method estimates the cell type constitution in the compound RNA-Seq

108 samples using relevant single cell data as a training source. Genes used for deconvolution were

109 automatically selected from the single cell data based on their information richness. Uniquely,

110 it uses an adaptively learning approach to estimate gene-wise scaling factors, addressing the

111 issue that each platform impact genes differently. AdRoit further makes use of regularization to

112 avoid collinearity among closely related cell subtypes that are common in complex tissues. Over 
113 a set of comprehensive benchmarking data with a varying cell composition complexity, AdRoit

114 showed superior sensitivity and specificity to other existing methods. Applications to real RNA-

115 Seq bulk data and spatial transcriptomics data revealed strong and expected biologically

116 relevant information. We believe AdRoit offers an accurate and robust tool for cell type

117 deconvolution and will enhance the value of bulk RNA-Seq and the spatial transcriptome

118 profiling.

\section{Results}

121 Overview of the AdRoit framework

122 AdRoit estimates the proportions of cell types from transcriptome data of a mixed cell

123 population including but not limited to bulk RNA-Seq and spatial transcriptome. It directly

124 models the raw reads without normalization, preserving the difference in total amounts of RNA

125 transcript in different cell types. The method utilizes, as a reference, the relevant pre-existing

126 scRNA-Seq data with cell identity annotation. It selects informative genes, estimates the mean

127 and dispersion of the expression of selected genes per cell type, and constructs a weighted

128 regularized linear model to infer percent combinations (Fig. 1a). Because sequencing platform

129 bias may impact genes differently ${ }^{15,34,35}$, a uniform scaling factor for all genes does not

130 sufficiently eliminate such bias. A key innovation of AdRoit is that it uniquely adopts an adaptive

131 learning approach, where the bias is first estimated for each gene, then adjusted such that

132 more biased genes are corrected with a larger scaling factor (Fig. 1b). 

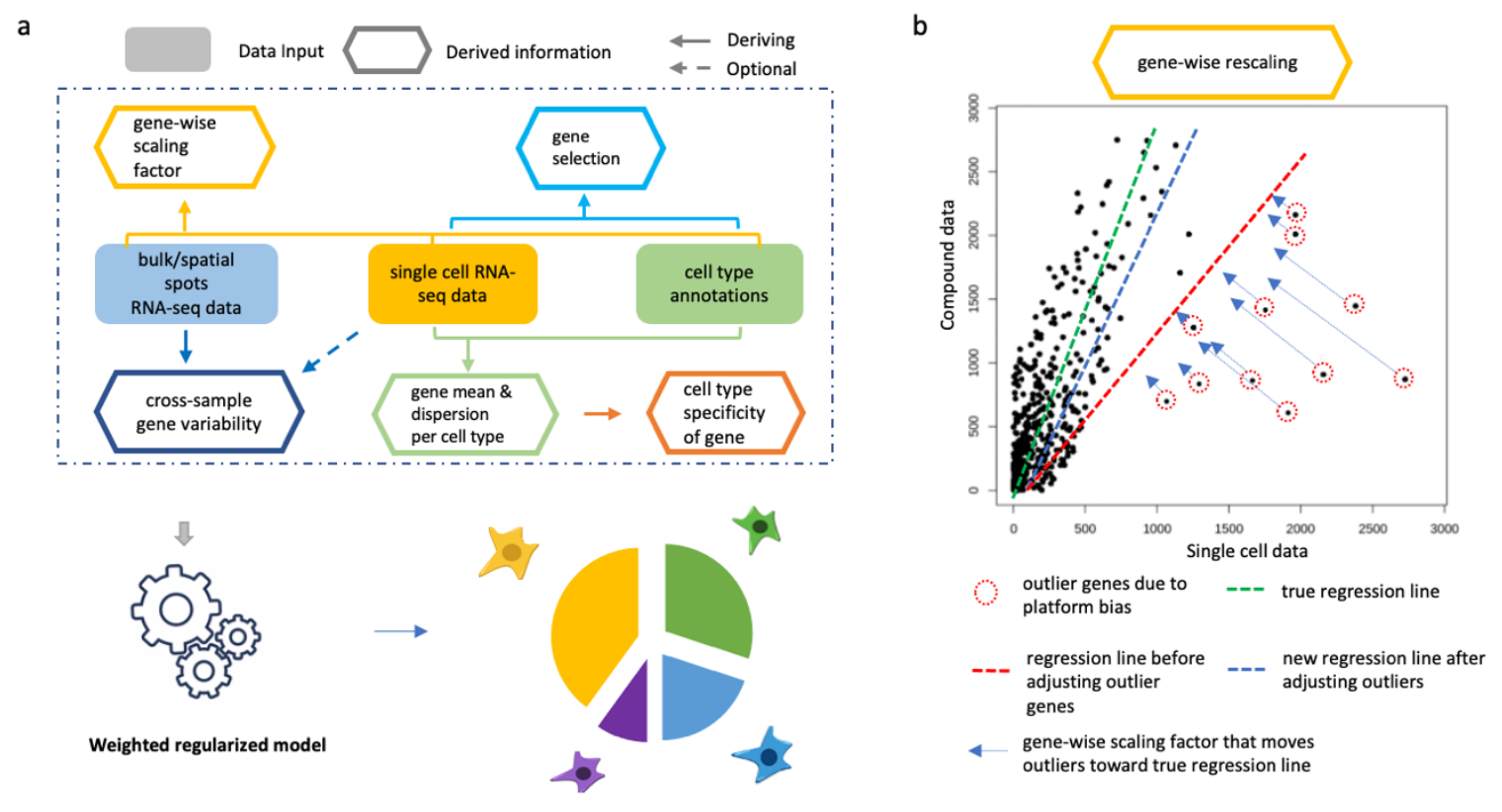

Fig. 1

134 AdRoit considers a comprehensive set of other relevant factors including the cross-sample

135 variability and the cell type specificity of genes, as well as the collinearity of expression profiles

136 among closely related cell types. The cross-sample variability of a gene is the variation of its

137 expression in the same cell type across multiple bulk samples. It shall be distinguished from the expression change in the gene over different cell types. AdRoit decreases the weight of genes

139 with high cross-sample variability whilst increasing the weight of those with an expression

140 highly specific to certain cell types. The definition of cross-sample variability and cell type

141 specificity also accounts for the overdispersed nature of counts data. Lastly, AdRoit adopts a

142 linear model to ensure the interpretability of the coefficients. At the same time, AdRoit includes

143 a regularization term to minimize the impact of statistical collinearity. Each of the factors 
144 contributes an indispensable part to AdRoit, leading to an accurate and robust deconvolution

145 method for inferring complex cell compositions.

147 To evaluate the performance, we compared AdRoit with NNLS ${ }^{18,36}$, MuSiC ${ }^{22}$, Bisque ${ }^{37}$, and

148 SPOTlight ${ }^{38}$ for bulk data deconvolution, and Stereoscope ${ }^{23}$, Cell2location ${ }^{39}$, and SPOTlight ${ }^{38}$ for

149 spatial transcriptomics data deconvolution. When evaluating the algorithms, a common

150 practice is to pool the single cell data to synthesize a "bulk" sample with the known ground

151 truth of the cell composition. We measured the performance by comparing the estimated cell

152 proportions with true proportions using four metrics: mean absolute difference (mAD), root

153 mean square deviation (RMSD), and two correlation statistics (i.e., Pearson and Spearman).

154 While Pearson reflects linearity, Spearman measures whether the estimated results and the

155 ground truth are monotonically related, even if their relationship is not linear, which avoids the

156 artificial high linearity scores driven by outliers when the majority of estimates are small. Thus,

157 both correlations statistics were included. Good estimations feature low mAD and RMSD along

158 with high correlation statistics. When estimating cell proportions for a synthetic sample, cells

159 from this sample were excluded from the input single cell reference (i.e., leave-one-out) to

160 avoid overfitting. We further applied AdRoit to real bulk RNA-Seq data and validated the results

161 by available RNA fluorescence in-situ hybridization (RNA-FISH) data. The estimates were further

162 confirmed by the biological knowledge of human pancreatic islets. We also used AdRoit to map

163 cell types within spatial spots, and the accuracy was verified by in-situ hybridization (ISH)

164 images from Allen mouse brain atlas ${ }^{40}$. 


\section{AdRoit excels in both simple and complex cell constitutions}

167 We started with a simple human pancreatic islets data set that contains 1492 cells and four

168 distinct endocrine cell types (Alpha, Beta, Delta, and PP cells) ${ }^{41}$ (Supplementary Fig. 1a;

169 Supplementary Table 1). The synthesized bulk data were constructed by mixing the single cell

170 data at known proportions. All methods except SPOTlight achieved satisfactory performance

171 according to the evaluation metrics, AdRoit performed the best as reflected by scatterplots of

172 estimated proportion vs. true proportion (Supplementary Fig. 1b, Supplementary Table 2). It

173 had the lowest mAD and RMSD, and the highest correlation values (MuSiC's correlations are

174 comparable) among the five methods tested (Supplementary Fig. 1c). Given that MuSiC is a

175 weighted NNLS and consistently showed an improved accuracy here and in the original

176 publication ${ }^{22}$, we excluded NNLS from further evaluation hereafter.

178 We then tested the methods on a complex tissue - human trabecular meshwork (TM). We

179 acquired published single cell data that contains 8758 cells and 12 cell types from 8 donors ${ }^{42}$.

180 The data include 3 similar types of endothelial cells, 2 types of Schwann cells, and 2 types of TM

181 cells (Supplementary Fig. 2; Supplementary Table 3). Cells from each donor were pooled as

182 individual synthetic bulk samples. The cell type proportions vary from $<1 \%$ to $43 \%$. These

183 proportions were the ground truth cell composition and were compared head-to-head with the

184 estimated proportions inferred by four methods. For each synthetic bulk sample, estimations

185 were performed using a reference built from cells of remaining donors (i.e., leaving-one-out). In

186 each of the 8 samples, the estimates made by AdRoit best approximated the true proportions.

187 In particular, AdRoit had the lowest mAD (0.016) and RMSD (0.025), and the highest correlation 
values (Pearson $=0.97 ;$ Spearman $=0.94$ ) among the methods (Fig. 2a). We further assessed the

192 (Supplementary Fig. 3, Supplementary Table 4).

a

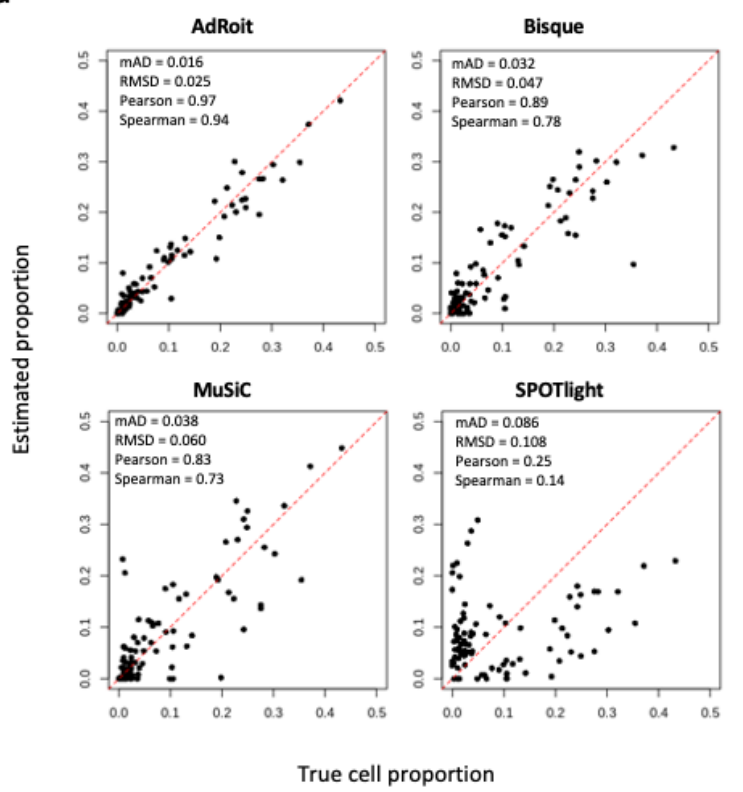

b

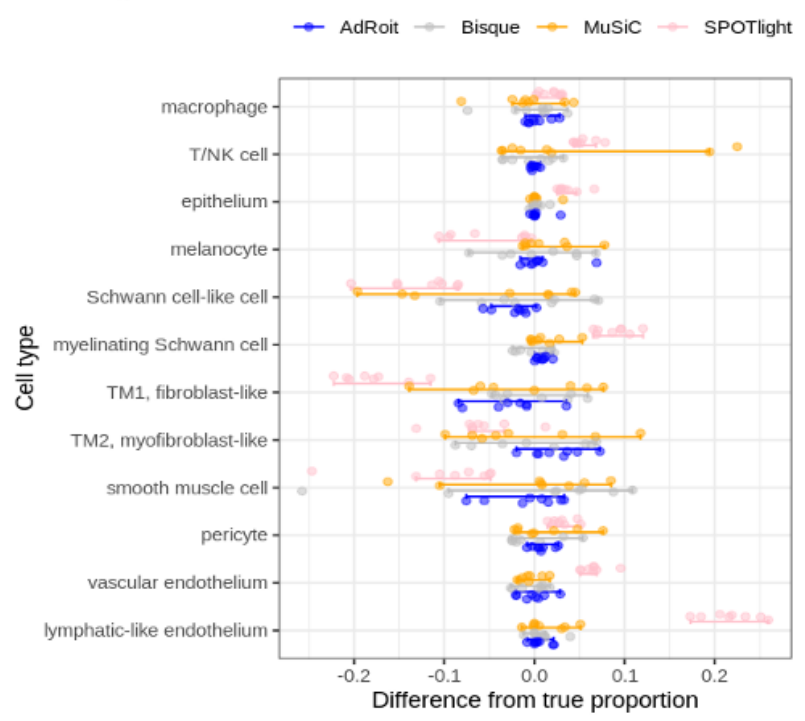

Fig. 2

194

195

196

197

198

\section{AdRoit has better sensitivity and specificity}

To assess the level of granularity that AdRoit can achieve when deconvoluting similar cell types, we used well-studied, closely related immune cells from myeloid lineage and lymphoid lineage.

We obtained $\mathrm{B}$ cells, naïve $\mathrm{CD} 4^{+} \mathrm{T}$ cells, memory $\mathrm{CD} 4^{+} \mathrm{T}$ cells, $\mathrm{CD} 8^{+} \mathrm{T}$ cells, natural killer (NK) cells, dendritic cells (DC), CD14 ${ }^{+}$monocytes, FCGR3A ${ }^{+}$monocytes, and platelets from a public release of the human peripheral blood mononuclear cell (PBMC) single cells by 10x Genomics (see also Data Availability). We simulated bulk samples that each contained a mixture of 
201 myeloid lineage or lymphoid lineage cell types. Such a sample was created by randomly

202 selecting cells from a given set of cell types and mixing them according to their predefined

203 percentages. We repeated this procedure 100 times for a series of mixing proportions among

204 myeloid or T lymphoid cells. The bulk mixtures of CD14 ${ }^{+}$monocytes, FCGR3A ${ }^{+}$monocytes and

205 dendritic cells (DC) followed three schemes of proportions: 0.33:0.33:0.33 (mix0), 0.1:0.45:0.45

206 (mix1) and 0.1:0.3:0.6 (mix2). The same ratios were applied to the mixtures of naïve $\mathrm{CD}^{+} \mathrm{T}$,

207 memory $\mathrm{CD}^{+} \mathrm{T}$ and $\mathrm{CD} 8^{+}$cells. All the nine cell types in the PBMC data were used to build the

208 reference for the deconvolution. AdRoit accurately estimated the known percentages in all

209 cases (Fig. 3a, Supplementary Table 5). In addition, there were no noticeable false positive

210 predictions of any excluded cell types. These results highlight that AdRoit can capture the fine

211 difference among similar cell types and quantitatively distinguish them. 

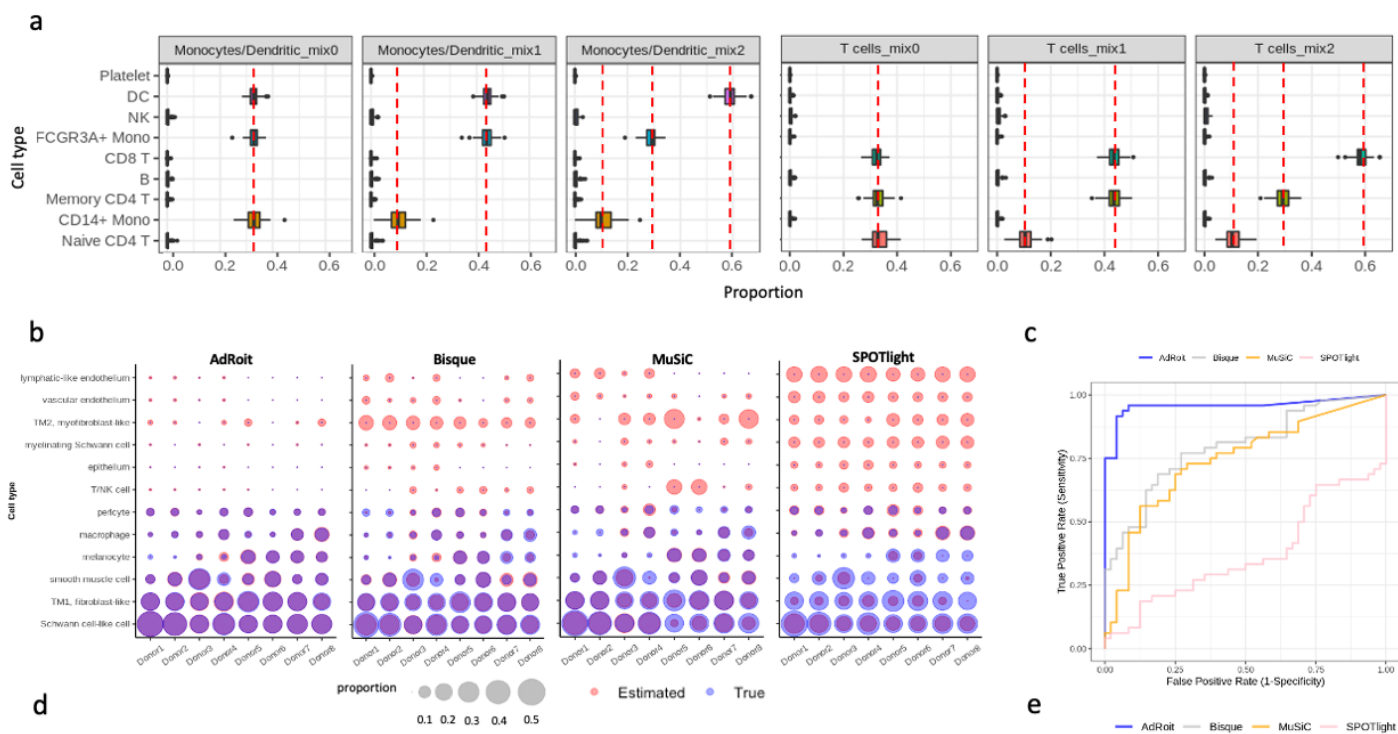

C

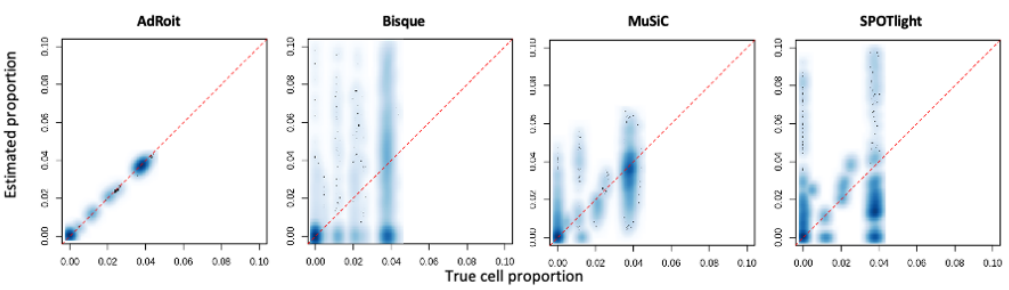

Fig. 3

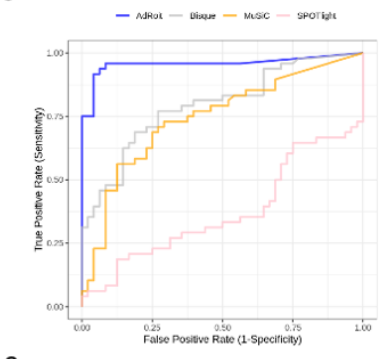

e

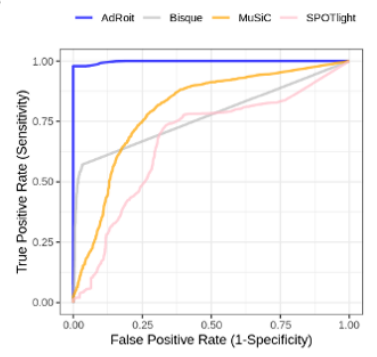

We next systematically benchmarked the sensitivity and specificity of each of the algorithms. In

214 the context of the cell type deconvolution, a false negative occurs when the proportion of an

215 existing cell type is predicted to be zero (or below a given threshold). Conversely, a non-zero

216 prediction (or above a given threshold) of an absent cell type results in a false positive. False

217 negatives and false positives measure the sensitivity and specificity of a deconvolution

218 algorithm, respectively. Both quantities are crucial to establish the utility of the algorithm.

219 Particularly, in real-world applications, it is often difficult to know a prior what cell types exist in

220 a bulk sample, users may inform the algorithm to consider more possible cell types than what

221 actually exist in the sample. False positive predictions in this situation would make the

222 algorithm unusable. 
224 We designed a simulation to test sensitivity and specificity. we selected 6 out of the 12 human

225 trabecular meshwork cell types, i.e., Schwann-cell like cell, TM1, smooth muscle cell,

226 melanocyte, macrophage, and pericyte, from each donor sample and pooled them within that

227 sample to synthesize 8 new bulk samples. The unselected 6 cell types were considered absent

228 in the bulk samples. Some cell types selected highly resembled those in absence, challenging

229 the programs to pinpoint the right cell type present in the bulk among similar candidates. We

230 provided the full list of 12 single cell types as the reference to the programs to estimate the cell

231 type proportions. Consistently across 8 samples, AdRoit had the most accurate estimates for

232 the 6 present cell types, and 0 or close to 0 estimated values for the non-existing cell types in

233 the simulated bulk data. MuSiC, Bisque and SPOTlight were notably less accurate on the 6

234 selected cell types, as well as they had many non-negligible values for the 6 cell types excluded

235 in the 8 synthetic samples (Fig. 3b, Supplementary Table 6). For example, smooth muscle cells

236 accounted for $\sim 14 \%$ in donor 4 but were largely missed $(\sim 0.03 \%)$ by MuSiC. We noted that TM2

237 had false non-zero estimates from all methods though not included. This is because TM2 is

238 easily mistaken as TM1 due to their high similarity ${ }^{42}$. Nonetheless, AdRoit's estimates of TM2

239 were consistently low across samples (<1\% for 44 out of 48 estimates), while the other

240 methods had significantly larger estimates of TM2 that occasionally even exceeded the TM1

241 estimates. For a systematic comparison, we constructed the receiver operating characteristic

242 (ROC) curve by varying the threshold of detection (i.e. a cutoff below which the cell type was

243 deemed undetected) (Fig. 3c). AdRoit had a significantly higher area under the curve (AUC) than 
244 the other methods (AdRoit: 0.95, Bisque: 0.79, MuSiC: 0.74, SPOTlight: 0.37), implying both

245 better sensitivity and specificity.

246

247 As complex tissues often contain several tens of different cell types, we continued the

248 evaluation of the sensitivity and specificity in more complicated cell mixtures. We utilized the

249 published mouse brain single cell atlas by Zeisel et al that contains a comprehensive set of

250 neuronal and supporting cells ${ }^{32}$. Without loss of generality, we consolidated the original

251 annotation into 46 major cell types (Methods, Supplementary Table 7). To synthesize a bulk

252 mixture, we randomly selected 30 cell types and pooled all their associated cells. This

253 procedure was repeated independently 100 times for thorough coverage of different cell type

254 combinations. The deconvolution was performed using all 46 cell types as the reference. As

255 illustrated in Fig. 3d, AdRoit was able to estimate the proportions closely consistent with the

256 ground truth while the other three tools made less satisfactory predictions (see also

257 Supplementary Table 8). The ROC curve further indicates that AdRoit has the best sensitivity

258 and specificity given its highest AUC (Fig. 3e; AdRoit: 0.99, Bisque: 0.77, MuSiC: 0.80, SPOTlight:

259 0.67). These observations convince us that AdRoit offers a leading performance on highly

260 complex cell constitutions.

261

262 AdRoit outperforms in deconvoluting closely related cell subtypes

263 To further evaluate AdRoit when multiple highly homologous subtypes of cells are present in a

264 complex tissue, we performed scRNA-Seq experiment on mouse lumbar dorsal root ganglion

265 (DRG) from five mice. Following the standard analysis pipeline (Methods), we obtained 3352 
266 single cells after quality control procedures. After clustering and annotation, we discovered 14

267 cell types including multiple subtypes of neuronal cells (Fig. 4a, Supplementary Table 9). The

268 heatmap of the top marker genes showed distinct patterns of the major cell types as well as

269 similar patterns of the subtypes (Supplementary Fig. 4a), with the cell type proportions varying

270 from $0.5 \%$ to $33.71 \%$ (Supplementary Fig. 4b). These 14 cell types include 3 subtypes of

271 neurofilament containing neurons (i.e., NF_Calb1, NF_Pvalb, NF_Ntrk2.Necab2), 3 subtypes of

272 non-peptidergic neurons (i.e., NP_Nts, NP_Mrgpra3, NP_Mrgprd), and 5 subtypes of

273 peptidergic neurons (i.e., PEP1_Dcn, PEP1_S100a11.TagIn2, PEP1_Slc7a3.Sstr2,

274 PEP2_Htr3a.Sema5a, PEP3_Trpm8). Also discovered were tyrosine hydroxylase containing

275 neurons (Th), satellite glia, and endothelial cells. Such complex compositions formed a

276 challenging testing ground for evaluating the ability to distinguish closely related cell types. We

277 again performed the leave-one-out deconvolution on five synthesized bulk samples.

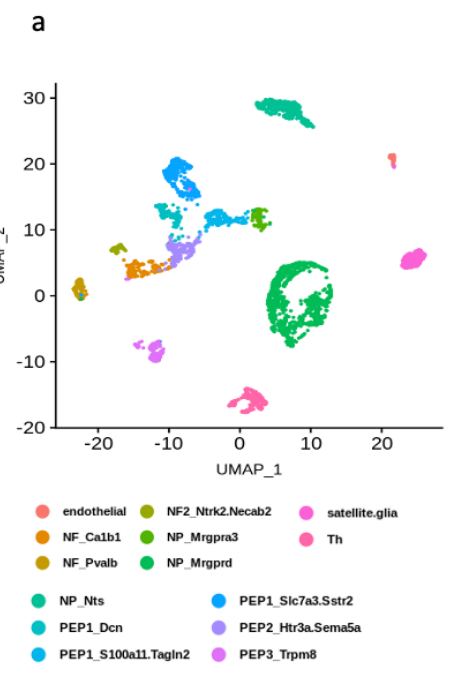

b

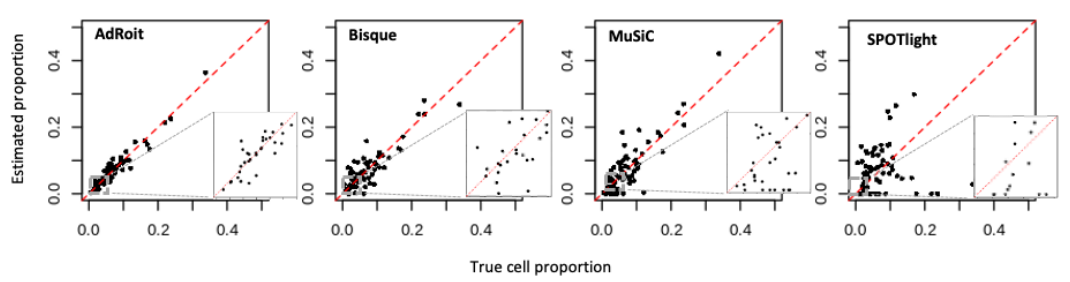

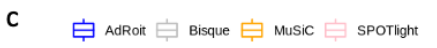
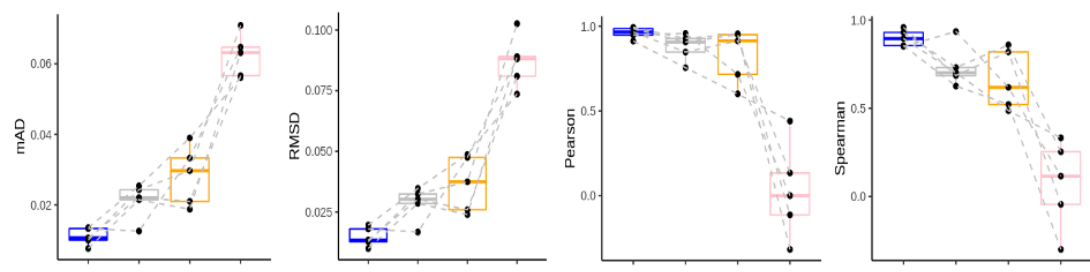
279 AdRoit had highly accurate estimations on all cell subtypes across samples (Fig. 4b). Worth

280 noting, for the rare cell types that account for less than 5\%, AdRoit still had a good estimation

281 that is close to the true proportions and never missed a single cell type, showing that AdRoit is

282 very robust on rare cell types. For example, $0.51 \%$ endothelial cells were predicted to be $0.35 \%$,

283 and 1.05\% NF2_Ntrk2.Necab2 cells were predicted to be 0.85\% (Supplementary Fig. 5,

284 Supplementary Table 10). Conversely, Bisque, MuSiC, and SPOTlight were notably less accurate,

285 especially for the cell types less than 5\%, and all three missed multiple cell types including some

286 large clusters accounting for $\sim 10 \%$ of the cells (PEP1_Slc7a3.Sstr2 cells of Sample5). We

287 continued to examine how much the variability of the estimates was in each sample. We

288 computed the same 4 metrics used previously to evaluate the performance on each of the 5

289 synthetic samples and compared them head-to-head among the algorithms. This fine

290 comparison showed AdRoit significantly outperformed on nearly every sample (Fig. 4c).

291 Moreover, the performance metrics of AdRoit were highly consistent across samples with the

292 lowest variability among all four methods.

293

294 AdRoit excels on spatial transcriptomics data

295 Given the promising performance on complex tissues, we moved forward to test AdRoit's

296 applicability to spatial transcriptome data. Spatial transcriptomics data differs from bulk RNA-

297 Seq data in that each spot only contains transcripts from a handful of cells $(3-30)^{12}$. Some of the

298 spots contain multiple cells of the same type, while others may have heterogeneous cell types

299 at varying mixing percentages (e.g., spatial spots at the boundary of different cell types). As the

300 spot is a pool of only a few cells, the variations across spatial spots are expected to be greater 
301 than the changes in bulk samples, imposing an additional challenge for deconvolution. We

302 simulated a large number of spatial spots ( 3200 in total) by using sampled cells from the DRG

303 single cell data above (Methods), then compared AdRoit with Stereoscope, Cell2location, and

304 SPOTlight over a range of simulation scenarios.

305

306 We first tested whether the methods could correctly infer a single cell type when the spots

307 contain cells from that same type. For each of the 14 cell types from DRG, we sampled 10 cells

308 and pooled them to form a spatial spot. We repeated the simulation 100 times for robust

309 testing, then used the full set of 14 cell types as reference to deconvolute the 1400 simulated

310 spots. All methods except SPOTlight were able to identify the correct cell types with high

311 accuracy on the target cell types (i.e., percentages close to 1) and comparably low estimated

312 values (i.e., percentages close to 0 ) for other cell types excluded from the

313 simulation(Supplementary Fig. 6, Supplementary Table 11).

315 We then proceeded to a more difficult scenario where we sampled cells from the 5 PEP neuron

316 subtypes and mixed them. We created three simulation schemes for a comprehensive

317 evaluation: 1) 5 PEP subtypes had the same percent of 0.2 ; 2) PEP1_Dcn was 0.1 and the other

3184 were 0.225; 3) PEP1_S100a11.TagIn2 and PEPE1_Den were 0.1, PEP2_Htr3a.Sema5a and

319 PEP1_Slc7a3.Sstr2 were 0.2, and PEP3_Trpm8 was 0.4. Again, each simulation scheme was

320 repeated 100 times. Under each scheme, the estimates by AdRoit consistently centered around

321 the true proportions while the predictions of irrelevant cell types remained close to 0 (Fig. 5a,

322 Supplementary Table 11). In comparison, Cell2location systematically overestimated the 
PEP1_Slc7a3.Sstr2 by about $2.5 \%$. Stereoscope more notably overestimated

a
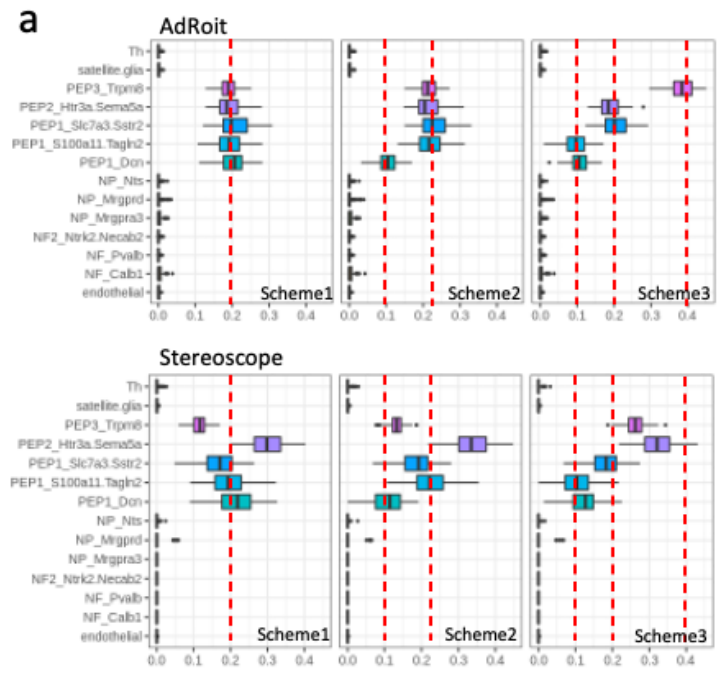

b

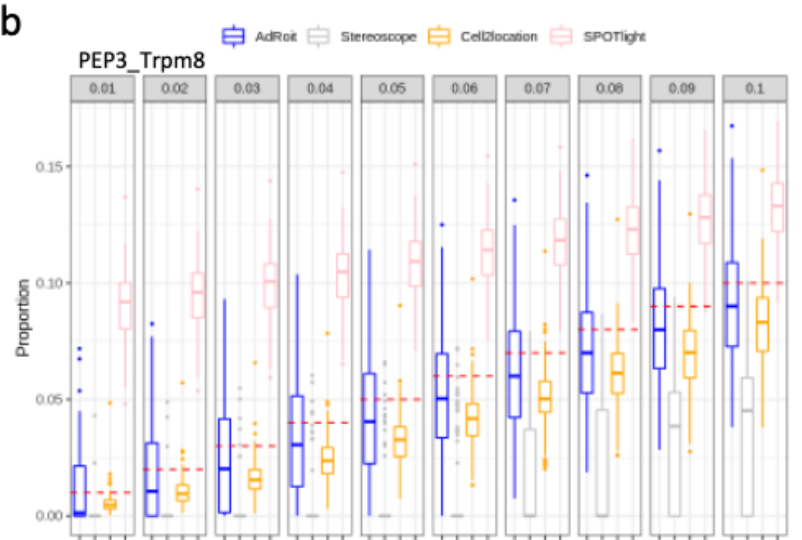

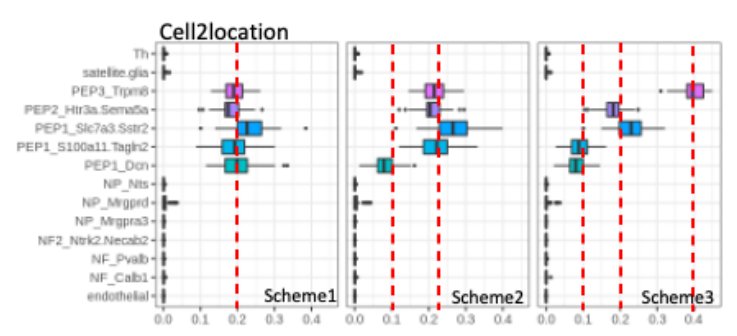

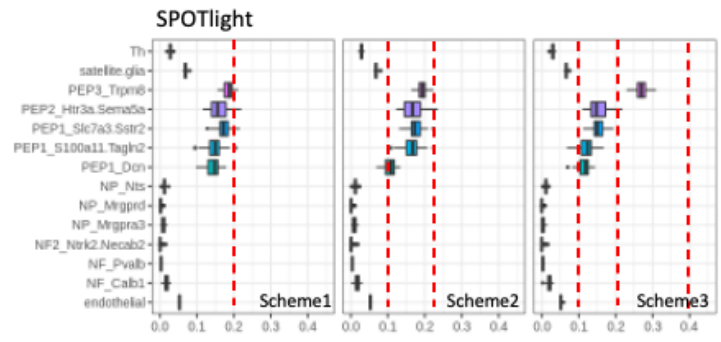

C
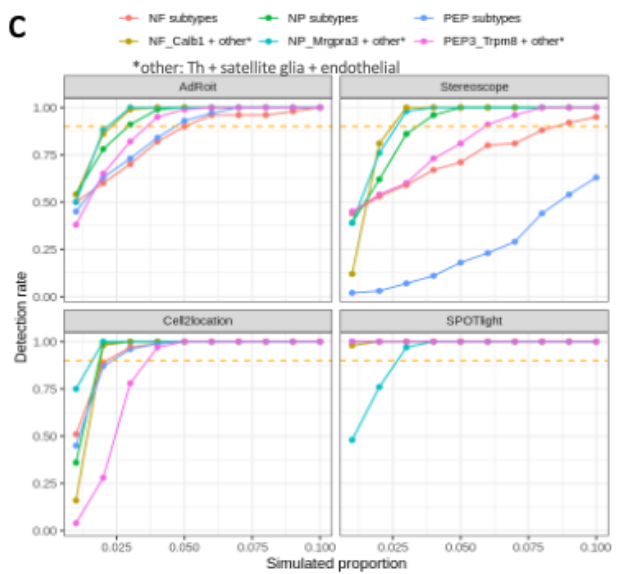

Fig. 5

328 We expanded the simulated spatial spots to the mixture of 3 NP cell subtypes and the mixture of 3 NF subtypes. In addition, we blended either NP_Mrgpra3 cells, NF_Calb1 cells, or PEP3_Trpm8 cells with three other cell types (i.e., Th, satellite glia and endothelial). For all

331 these simulated spatial spots, AdRoit's estimates consistently centered around the true 
332 proportions, whereas the other methods deviated by different degrees in most of the simulated

333 schemes (Supplementary Fig. 7, Supplementary Table 11). We speculate one important reason

334 for the underperformance of some methods such as Stereoscope is that they normalize the

335 total UMI counts to the same value for all cells. However, in real world, different cell types

336 unlikely have the same amount of RNA transcripts (e.g. immune cells have about 10 fold fewer

337 total UMIs than the neurons). Our simulation pooled the cells by adding up the raw UMI counts

338 per gene, which we believe best mimics the real data. Normalizing the data by total UMI not

339 only eliminates this factual difference between cell types but may distort the data from the

340 assumed negative binomial distribution. AdRoit avoids this problem by model the raw UMI

341 counts.

343 Next, we asked how sensitive the methods are in detecting rare cell populations. We simulated

344 mixtures of 3 PEP subtypes (i.e., PEP1_Slc7a3.Sstr2, PEP2_Htr3a.Sema5a, and PEP3_Trpm8)

345 wherein the percentage of PEP3_Trpm8 cells varied from 0.01 to 0.1 by 0.01 , and the other two

346 cell types sharing the remaining percentage equally (Methods). At each given mixing ratio, the

347 simulation was repeated 100 times. We then checked how accurately the concentration of

348 PEP3_Trpm8 cells was estimated. The medians of AdRoit's estimates were closest to the true

349 proportions (Fig. 5b, red dashed lines) among all 4 methods, followed by Cell2location.

350 Stereoscope's estimates were systematically lower than the true values and failed to detect the

351 PEP3_Trpm8 cells when the simulated proportion was below 0.06. On the other hand,

352 SPOTlight constantly overestimated the presence of PEP3_Trpm8 cells. The median of its

353 predictions remained around $9 \%$ even when the true population was at $1 \%$. Such observations 
354 are consistent with the frequent false positives made by SPOTlight when deconvoluting the

355 simulated bulk samples (Fig. 3b). This comparison implied AdRoit is more advantageous in

356 detecting low percent cells. For a complete evaluation, we replicated the comparison using 5

357 additional sets of cell mixtures: NF_Calb1 with NF_Pvalb and NF2_Ntrk2.Necab2 (NP subtypes);

358 NP_Mrgpra3 with NP_Mrgprd and NP_Nts (NP subtypes); NF_Calb1 with Th, satellite glia and

359 endothelial (NF_Calb1 + others); NP_Mrgpra3 with Th, satellite glia and endothelial

360 (NP_Mrgpra3 + others); and PEP_Trpm8 with Th, satellite glia and endothelial (PEP_Trpm8 +

361 others). In each set, the first cell type mentioned was considered rare with its percent varied

362 from 0.01 to 0.1 . The rest cell types shared the remaining proportion evenly. At each given

363 percent of the rare cell type, we computed how many times out of 100 the rare cell component

364 was detected (estimation $>0.005$ ). AdRoit had systematically high detection rates especially

365 when the percent is below 3\%. (Fig. 5c, Supplementary Table 12). Note that the apparent high

366 detection rates in SPOTlight were merely the result of its high false positive estimates (Fig. 3b

367 and Fig. 5b). The detection sensitivity of Cell2location was comparable to that of AdRoit.

368 Notably, given a rare cell population of 5\%, both AdRoit and Cell2location achieved a detection

369 rate $>90 \%$ under all simulation schemes, making them powerful tools to uncover rare cells.

\section{Application to real bulk RNA-Seq data of human pancreatic islets}

372 Though using synthetic bulk data based on the mixing of single cells is a useful benchmarking

373 strategy, the bulk and single cell RNA-Seq often use distinct RNA library preparations and

374 sequencing protocols. The capability of a method to deconvolute real bulk samples shall be

375 addressed to ensure it is useful in real-world applications. We acquired 70 real human 
376 pancreatic islets bulk samples from published studies ${ }^{41,43,44}$ (Supplementary Table 13) and used

377 single cell data of the same tissue ${ }^{41}$ as the reference to infer the percentages of 4 endocrine cell

378 types (i.e., Alpha, Beta, Delta, PP). The 70 bulk samples were collected from 39 distinct donors,

379 including 26 healthy donors, and 13 donors with type 2 diabetes (T2D). Each donor contributed

3801 to 5 bulk RNA sample repeats.

381

382 Replicates from the same donor are expected to have similar compositions and thus were used

383 to assess the reproducibility of the estimates from AdRoit. For all cell types, AdRoit had

384 consistent estimates for the same donors (Fig. 6a, Supplementary Table 14). The average

385 standard deviations did not exceed 1\% for all 4 cell types (i.e., Alpha: 0.010; Beta: 0.008; Delta:

386 0.004; PP: 0.002). To seek an independent validation, we obtained cell sorting results by RNA-

387 FISH for 4 of the 39 donors $^{41}$ (Supplementary Table 15). The estimated cell proportions in the 4

388 samples agreed well with the percentages measured by RNA-FISH (Fig. 6b). The consistency

389 held for both major cells (Alpha and Beta) and the minor cells (Delta and PP). The observed

390 reproducibility and the success of the independent validation showed AdRoit is reliable in

391 deconvoluting real bulk RNA-Seq data. 


$$
\text { a }
$$


404 Application to mouse brain spatial transcriptomics

405 We lastly demonstrated an application to the real spatial transcriptomics data. Given that the

406 molecular architecture of brain tissues has been well studied, for this evaluation, we chose a set

407 of mouse brain spatial transcriptomics data generated by 10x genomics, containing 2703 spatial

408 spots. The consolidated version of the aforementioned mouse brain single cell atlas ${ }^{32}$ was used

409 as the reference for deconvolution (Methods).

410

411 The cell contents inferred by AdRoit per spot appear to accurately match the expected cell

412 types at that location (Supplementary Fig. 8, Supplementary Table 16). For example, the three

413 subtypes of cortex excitatory neurons each occupied a sub-area in the cerebral cortex region.

414 As another example, the shape of the hippocampal region was delineated by the estimated

415 percentages of dentate gyrus granule/excitatory neurons. As an independent validation, we

416 checked the consistency between the estimated cell types with the in-situ hybridization (ISH)

417 images from Allen mouse brain atlas ${ }^{52}$. We chose 4 genes highly expressed in 4 brain regions

418 respectively, i.e., Spink8 for the hippocampal field CA1; C1qI2 for the dentate gyrus; Clic6 for

419 the choroid plexus; and Synpo2 for the thalamus ${ }^{32}$. The spots enriched with the 4 indicative cell

420 types, hippocampal CA1 excitatory neuron type 2, dentate gyrus granule neuron type 2, choroid

421 plexus cell and thalamus excitatory neuron type 1, as mapped by AdRoit, precisely co-localized

422 with the regions that showed a strong signal of the 4 marker genes on the ISH images

423 respectively (Fig. 6d). This agreement confirmed that the spatial mapping of cell types by AdRoit

424 is reliable.

425 


\section{Computational efficiency}

427 Besides the accuracy and robustness, another major advantage of AdRoit is its magnitude

428 higher computational efficiency. AdRoit uses a two-step procedure to do the inference. The first

429 step prepares the reference using the single cell data where the per-gene means and

430 dispersions are estimated, and cell type specificity is subsequently computed. The built

431 reference can be saved and reused. We tested the running time on the reference construction

432 using the mouse brain single cell dataset discussed earlier that contains $\sim 15,000$ cells. It took

433 about 4.5 minutes on a CPU that has 24 cores (23 used for parallel computing). The second step

434 inputs the built reference and the compound RNA-Seq data and does the estimation.

435 Deconvoluting 2700 RNA-Seq samples of mixed cell populations took around 5 minutes.

436 Therefore, AdRoit in total took less than 10 minutes and $~ 3 \mathrm{~Gb}$ memory usage on a regular CPU.

437 As a comparison, MuSiC and Bisque took about 1 hour and 30 minutes on the same data using

438 the same CPU setup. SPOTlight took about 2 hours with the default parameters (cl_n $=100$, hvg

$439=3000$, ntop $=200$, transf $=$ "uv", method $=$ "nsNMF", min_cont $=0$ ). Stereoscope ran about 24

440 hours continuously with the published parameter setting (-scb 256 -sce 75000 -topn_genes

4415000 -ste 75000 -Ir 0.01 -stb 100 -scb 100) on a powerful V100 GPU with 80 cores and 16G

442 memory. The efficiency of Cell2location was comparable to Stereoscope, taking about 18 hours

443 on the V100 GPU (posterior n_sample $=1000$, n_iteration $=10000$, selection_specificity $=$

444 0.45). These methods can be prohibitive when seeking a quick turnaround.

\section{Discussion}


447 In this work we have demonstrated that AdRoit is capable of deconvoluting the cell

448 compositions from the compound RNA-Seq data with a leading accuracy, measured by the

449 consistency between the true and predicted cell proportions. Its advantage over the existing

450 state-of-the-art methods was verified over a wide range of use cases. In particular, AdRoit

451 excelled in complex tissues composed of more than ten different cell types with a wide range of

452 cell proportions (e.g., trabecular meshwork, mouse brain and dorsal root ganglion). In these

453 cases, AdRoit performed significantly better than the comparators on deconvoluting bulk RNA-

454 Seq data. AdRoit is also more accurate and sensitive than tools specifically designed for

455 deconvoluting spatial transcriptomics spots, especially in detecting low percent rare cells.

456 Previous benchmarking often assumed the types of cells in the synthetic bulk data are not more

457 or less than the cell types collected in the reference, and thus the only unknown was the

458 proportion of each cell type. This assumption may not hold. Missing existing cell types or false

459 predictions of non-existing ones can hinder the utility of an algorithm. Thus, besides the overall

460 accuracy, we also examined the sensitivity and specificity of the algorithms. We observed a

461 superior sensitivity and specificity in AdRoit, an important leverage for its usage in practice.

462

463 The reference single cell data used by AdRoit came from different platforms, such as the $10 \mathrm{x}$

464 Genomics Chromium Instrument (the mouse dorsal root ganglion), and the Fluidigm C1 system

465 (the human pancreatic islets data). AdRoit consistently exhibited excellent performance across

466 all benchmarking datasets independent of their single cell sequencing technology platforms.

467 More importantly, this statement holds not only for deconvoluting the synthesized bulk data,

468 but also for the real bulk RNA-Seq data. The latter typically does not apply the unique molecular 
469 barcoding and requires a significantly different cDNA amplification procedure from what is used

470 in the single cell RNA-Seq (Methods). Moreover, the sequencing depth, read mapping and gene

471 expression quantification are dissimilar as well. The fact that AdRoit accurately dissected the

472 cell compositions in the real bulk samples based on the single cell reference data further

473 supports its cross-platform applicability.

474

475 We attribute the power of AdRoit to its comprehensive modeling of relevant factors. Firstly, we

476 think a common rescaling factor is insufficient to correct the platform difference between

477 single cells and the compound data. Rather, the impact of platform difference on genes is quite

478 different and not linearly scaled. Correcting such differences entails rescaling factors specifically

479 tailored to each gene. AdRoit uses an adaptive learning approach to estimate such gene-wise

480 correcting factors and does the correction in a unified model. In addition, the contribution of a

481 gene in a cell type to the loss function is jointly weighted by its specificity and variability in a cell

482 type, where specificity and variability are defined in a way accounting for the overdispersion

483 property of counts data. Our observations over the multiple benchmarking data sets also show

484 that the coexistence of similar cell types may have induced a collinearity condition that

485 negatively impacted the regression-based methods developed by others. Being able to alleviate

486 this problem gives AdRoit an edge. All these factors help AdRoit to distinguish similar cell

487 clusters while remaining sensitive enough to separate rare cell types.

489 Technically, the input profiles of individual cell types to AdRoit need not come from the single

490 cell RNA-Seq. Bulk RNA-Seq profiles of individual isolated cell types can be used as well. 
491 Nevertheless, using single cell RNA-Seq data as the reference has a few key advantages. It is a

492 high throughput approach wherein multiple cell types can be interrogated simultaneously. Prior

493 knowledge of the cell types in presence as well as their specific gene markers are not required,

494 which allows novel cell types to be identified. Although detection of lowly expressed genes has

495 been a challenge for the single cell RNA-Seq, significant enhancements have been

496 demonstrated. For example, the number of detectable genes currently can reach an order of

49710,000 per cell and keeps improving ${ }^{53}$. As AdRoit focuses on the informative genes whose

498 expressions are generally high, the detection limit of the single cell RNA-Seq does not impose a

499 significant drawback. Indeed, given the single cell reference profiles, AdRoit successfully

500 deconvoluted the real bulk RNA-Seq data and spatial transcriptomics data. The results suggest

501 that, besides enriching our understanding of the bulk transcriptome data, AdRoit can leverage

502 the usage of the vast amount and continuously growing single cell data as well.

503

504 AdRoit is a reference-based deconvolution algorithm. A comprehensive collection of the

505 possible cell components is important. However, completeness may not always be guaranteed.

506 Even with the single cell acquisition that is independent of prior knowledge, rare and/or fragile

507 cell types may not survive through the capture procedure and hence are excluded. It is also

508 difficult to generate a solid reference profile for cells that are versatile from sample to sample

509 (e.g., tumor cells). Currently AdRoit deals implicitly with the components unknown to the

510 reference. If an unknown cell type reassembles one of the referenced ones, it may be

511 considered as part of the known cell type and their joint population is predicted. Such an

512 outcome is acceptable as treating two similar cell types as one is still biologically meaningful 
513 although the resolution of the system may be compromised. If the unknown component is

514 dissimilar to all the known ones, it will be ignored by AdRoit because its representative markers

515 are unlikely among the top weighted genes associated with the known components. At the

516 same time, the distinct component is expected to have a unique gene expression pattern and

517 thus unlikely to interfere significantly with the gene expressions from the known cell types.

518 Therefore, AdRoit essentially deconvolutes the relative populations among the known cell

519 components. For example, AdRoit was able to correctly uncover the populations of 4 endocrine

520 cell types from the human islet bulk data despite the absence of many other cell types such as

521 macrophages, Schwann cells and endothelial cells in the input single cell reference ${ }^{20}$. Although

522 under such a circumstance, the absolute percentages of the cells remain obscure, we expect

523 their relative proportions can be studied and valuable. A future improvement is to explicitly

524 model the unknown cell types and estimate their percentages upon the signals in the

525 compound data that cannot be explained by the contribution from the known components.

526 AdRoit may also be coupled with other programs ${ }^{54}$ to output the deconvoluted cell type specific

527 transcriptome profiles. The inferred cell type proportions by AdRoit can serve as the input to

528 the downstream program and benefit the outcome with the reliability and accuracy

529 demonstrated in this work.

531 Methods

\section{Gene selection}

533 AdRoit selects genes that provide information about cell type identity, excluding non-

534 informative genes that potentially introduce noise. There are two ways for selecting such 
535 genes: 1) union of the genes whose expression is enriched in one or more cell types in the

536 single cell UMI count matrix. These genes are referred to as marker genes; 2 ) union of the

537 genes that vary the most across all the cells in the single cell UMI count matrix, referred to as

538 the highly variable genes. For marker genes, we recommend selecting the top 200 genes,

539 ranked by fold change, from each cell type for resolving complex compound transcriptome

540 data. Considering some genes may be enriched in more than one cell type, we further require

541 selected markers presenting in no more than 5 cell types to ensure specificity. We also suggest

542 selecting a minimum of 1000 total unique marker genes for an accurate estimation. If not

543 satisfied, one may consider expanding the number of top genes and/or loosen the statistical

544 significance cutoff.

545

546 AdRoit also offers the option to use highly variable genes. To avoid the selected highly variable

547 genes being dominated by large cell clusters, AdRoit first balances the cell types in the single

548 cell UMI count matrix by finding the median size (i.e., the number of cells) of all clusters, then

549 samples cells from each cluster to make its size equal to the median. Next, AdRoit computes the

550 variance of each gene across all the cells in the balanced single cell UMI matrix. The variance-

551 stabilizing transformation (VST) ${ }^{55}$ is applied to normalize the data prior to the variance

552 calculation. Genes with the top 2000 largest variances are then selected.

554 In both ways, mitochondria genes are excluded as their expression does not have information

555 of cell identity. The results shown in the current paper were based on the marker genes derived 
556 as described above. We also demonstrated that using the balanced highly variable genes

557 yielded comparably accurate estimations (Supplementary Fig. 9).

559 Estimate gene-wise mean and variance per cell type

560 Modeling single cell RNA-Seq data is challenging due to cell heterogeneity, technical sensitivity,

561 and noise. Some genes may not be detected by chance, while others may be found to be highly

562 dispersed. These factors can lead to excessive variability even within the same cell type. AdRoit

563 combats high noise and computational complexity by building models with estimated mean and

564 dispersion per gene per cell type. This strategy reduces the data complexity while preserves the 565 cell type specific information.

566

567 Although typical analyses of RNA-Seq data start with normalization, AdRoit does not do

568 normalization before the mean estimation. Performing a normalization across all cell types

569 forces every cell type to have the same amount of RNA transcripts, measured by the total

570 unique molecular identifier (UMI) counts per cell. However, different cell types can have

571 dramatically different amounts of transcripts. For example, the amount of RNA transcripts in

572 neuronal cells is about 5 times higher than that in glial cells. Thus, normalization can falsely

573 alter the relative abundance of cell types, misleading the estimation of cell type percentages.

574 To avoid this problem, AdRoit models the means using the raw UMI counts.

575

576 Studies have shown that UMI counts follow a negative binomial distribution ${ }^{56,57}$. We therefore

577 fit negative binomial distributions to genes of each cell type and build the model based on the 
579 of single cell UMI counts of gene $i \in 1, \ldots, I$ for all cells in cell type $k \in 1, \ldots, K . I$ is the number of

580 selected genes, and $K$ denotes the number of cell types in the single cell count matrix. The

581 distribution of $X_{i k}$ follows a negative binomial distribution,

$$
X_{i k} \sim N B\left(\lambda_{i k}, p_{i k}\right)
$$

583 where $\lambda_{i k}$ is the dispersion parameter of the gene $i$ in cell type $k$, and $p_{i k}$ is the success

584 probability, i.e., the probability of one observed UMI belonging to gene $i$ in cell type $k$. The two

585 parameters are estimated by MLE. The likelihood function is

586

$$
L H\left(\lambda_{i k}, p_{i k} \mid X_{i k}\right)=\prod_{i=1}^{n_{k}} f\left(X_{i k} \mid \lambda_{i k}, p_{i k}\right),
$$

587 where $n_{k}$ is the number of cells in cell type $k$, and $f$ is the probability mass function of the negative binomial distribution. The MLE estimates are then given by

$$
\left.\widehat{\left(\lambda_{l k}\right.}, \widehat{p_{l k}}\right)=\underset{\lambda_{i k}, p_{i k}}{\operatorname{argmax}} \operatorname{LH}\left(\lambda_{i k}, p_{i k} \mid X_{i k}\right)
$$

Once the success probability and the dispersion are estimated, the mean and the variance of

591 the negative binomial distribution can be computed numerically,

$$
\mu_{i k}=\frac{\widehat{\lambda_{l k}} \cdot \widehat{p_{l k}}}{1-\widehat{p_{l k}}}
$$

$$
\sigma_{i k}^{2}=\frac{\widehat{\lambda_{l k}} \cdot \widehat{p_{l k}}}{\left(1-\widehat{p_{l k}}\right)^{2}}
$$

594 Model fitting using MLE has been readily coded in many R packages. We choose the 'fitdist'

595 function from the 'fitdistrplus' package $^{58}$ for its fast computation speed and flexibility in 596 selecting distributions. The mean and variance estimations are done for each selected gene in 597 each cell type, resulting in a $I \times K$ matrix each. 


\section{Cell type specificity of genes}

600 Genes with a cell-type specific expression pattern better distinguish cell types, thus are more 601 important for resolving cell type composition. In line with this property, AdRoit weighs more 602 the genes with a higher cell type specificity. Highly specific genes usually have consistently high 603 expression and thus relatively low variance among cells within a cell type. To compute the cell

604 type specificity of a gene, we first identify the cell type in which the gene has the highest 605 expression (i.e., most expressed cell type), then defines the specificity of this gene as the mean-

606 to-variance ratio within that cell type. A high ratio renders a high weight to the gene in the 607 model. Here the mean and the variance refer to the estimated values from the negative 608 binomial model $\left(\mu_{i k}\right.$ and $\sigma_{i k}^{2}$ in eq. 4 and 5). Let $k^{\prime}$ be the index of the cell type that has the 609 highest mean expression of gene $i$,

$$
k^{\prime}=\underset{k}{\operatorname{argmax}}\left\{\mu_{i k} \mid k \in 1 \ldots K\right\}
$$

611 then the weight of the cell type specificity for gene $i$, denoted $w_{i}^{S}$, is given by,

$$
w_{i}^{S}=\frac{\mu_{i k^{\prime}}}{\sigma_{i k^{\prime}}^{2}}
$$

613 and it is computed for each gene in the set of selected genes.

\section{Cross-sample gene variability}

616 The variability of a gene contrasts with how stable a gene is across samples. The idea of

617 weighting genes based on their variability across samples is first explored by Wang et al ${ }^{22}$,

618 wherein the variability was defined as the cross-sample variance. By weighting down the high

619 variability genes, the authors achieved a great advantage over the traditional unweighted

method. Genes with a low cross-sample variability better represent the sample population, 
621 hence are more trustworthy for learning the cell composition. AdRoit incorporates the same

622 notion to weigh the importance of genes, however, it defines the variability more

623 comprehensively. AdRoit acknowledges the dependency between the gene expression level

624 and its variance and thus computes a variance-to-mean ratio (VMR) as the cross-sample

625 variability. Here the mean and the variance are computed across samples. The distribution of

626 the VMR values is less skewed than that of the variance alone. The VMR also circumvents

627 underweighting genes with a low expression, or overweighting genes highly dispersed. Let $Y_{i j}$

628 denote the counts for gene $i$ in sample $j \in 1, \ldots, J$, then

629

$$
Y_{i j} \sim N B\left(\lambda_{i j}, p_{i j}\right)
$$

630 where $\lambda_{i j}$ is the dispersion parameter of the gene $i$ in sample $j$, and $p_{i j}$ is the success

631 probability. Again, we use MLE to estimate $\widehat{\lambda_{l j}}$ and $\widehat{p_{l \jmath}}$, following which the cross-sample mean 632 and variance can be numerically computed:

$$
\mu_{i}^{S}=\frac{\widehat{\lambda_{l \jmath}} \cdot \widehat{p_{l j}}}{1-\widehat{p_{l \jmath}}}
$$

$$
\left(\sigma_{i}^{2}\right)^{S}=\frac{\widehat{\lambda_{l \jmath}} \cdot \widehat{p_{l j}}}{\left(1-\widehat{p_{l \jmath}}\right)^{2}}
$$

635 and the cross-sample variability VMR for gene $i$ is then defined as

$$
V M R_{i}=\frac{\left(\sigma_{i}^{2}\right)^{S}}{\mu_{i}^{S}}=\frac{1}{w_{i}^{C}}
$$

637 where $w_{i}^{C}$ is later used in the model. The cross-sample variability weight is computed for each 638 gene in the set of selected genes.

639 Typically, the RNA-Seq data of mixed cells to be deconvoluted include multiple samples to

640 control for biological variability. In this case, the VMR is computed as described above using the

641 cross-sample mean and variance of the gene. In case the RNA-Seq data to be deconvoluted lack 
642 multiple samples whereas the single cell reference contains replicates, AdRoit pools all cells per

643 replicate to synthesize multiple compound samples and subsequently estimate the VMRs with

644 them. If neither input has multiple repeats, AdRoit takes a bootstrapping approach to

645 subsample and pool cells from each cell type in the single cell reference several times to

646 generate multiple compound samples.

647

648

\section{Gene-wise scaling factor to correct the platform bias}

649 When comparing the RNA-Seq data of cell mixtures to the single cell data, a rescaling factor is

650 often used to account for the library size and platform difference. The existing methods adopt a

651 single rescaling factor for each sample, i.e., all genes in a sample are multiplied by the same

652 factor 22,23 . This operation is based on a strong assumption that the platform difference impacts

653 every gene equally and linearly scalable among different cell types, which hardly holds. In

654 addition, because linear models are sensitive to outliers, the estimation of cell proportions can

655 be steered away from the truth by genes that are significantly affected by the platform bias.

656 Therefore, applying a uniform scaling factor to all genes is inappropriate.

657

658 To overcome this problem, AdRoit estimates a gene-wise scaling factor per sample via an

659 adaptive learning strategy and rescales each gene with its respective scaling factor. To proceed,

660 we first input the mean gene expression from the mixture samples ( $\mu_{i}^{S}$ in eq. 9) and the

661 estimated means of each cell type from the single cell data ( $\mu_{i k}$ in eq. 4$)$, then apply a non-

662 negative least square regression (NNLS) to get a coarse estimation of the proportions of each

663 cell type, $\tau_{k}$. For each gene $i$, a predicted mean expression $\left(\sum_{k}^{K} \widehat{\tau_{k}} \mu_{i k}\right.$ in eq. 13) in the sample of 
664 mixture cells is computed as the weighted sum of the means of this gene in each cell type

665 wherein the weights are $\tau_{k}$. The regression equation is given by,

666

$$
\mu_{i}^{S}=A \cdot\left(\sum_{k}^{K} \tau_{k} \mu_{i k}+\varepsilon\right), \quad 0<\tau_{k}, \quad \sum_{k}^{K} \tau_{k}=1
$$

667

where $A$ is a constant to ensure the sum of $\tau_{k}$ 's is 1 and $\varepsilon$ is the error term. We use the 'nnls'

668 function in the 'nnls' package ${ }^{59}$ to estimate $\tau_{k}$ 's. Next, we calculate the ratio between the mean

669 expression from the mixture samples and the predicted counterpart, and define the gene-wise

670 rescaling factor as the logarithm of the ratio plus 1 ,

671

$$
r_{i}=\log \left(\frac{\mu_{i}^{S}}{\sum_{k}^{K} \widehat{\tau}_{k} \mu_{i k}}+1\right)
$$

672 Given the dispersed nature of count data, the logarithm of the ratio is a more appropriate

673 statistic as it results in relatively stable scaling factors. The addition of 1 avoids taking logarithm

674 on zeros. By multiplying the gene-wise rescaling factor, the genes affected more strongly by the 675 platform bias (i.e., "outliers") receive a larger correction (Fig. 1b).

676

\section{Weighted and regularized loss function for training}

678 AdRoit employs a non-negative least square regression model to infer the cell compositions in a

679 RNA-Seq sample of cell mixtures. The model (eq. 14) approximates the gene expression

680 observed in the mixture sample by the weighted (the weights are the mixing percentages) sum

681 of the cell type-specific gene expressions from the single cell reference, after correcting the 682 platform difference.

$$
y_{i j} \approx r_{i} \cdot \sum_{k}^{K} \beta_{k} \widehat{\mu_{l k}} \quad\left(\beta_{1}, \ldots, \beta_{K}>0\right)
$$

684 where $y_{i j}$ is the expression of gene $i$ in the mixture transcriptome sample $j$; the coefficient $\beta_{k}$ is 685 the mixing proportion for cell type $k$; $\widehat{\mu_{l k}}$ is the estimated mean expression of gene $i$ in cell type 
$686 k$ computed according to eq. $4 ; r_{i}$ is the gene-wise scaling factor computed by eq. 13 to correct

687 the bias due to the technology difference. To infer the $\beta_{k}$ coefficients, AdRoit uniquely

688 incorporates all the factors discussed in the previous sections. This is implemented in a

689 weighted sum-of-squared loss function $L$, where the weights consist of two components, $w_{i}^{C}$ in

690 eq. 7 and $w_{i}^{S}$ in eq. 11. Thus, a larger weight is given to the genes with a higher cell type

691 specificity and a lower cross-sample variability. In cases of complex tissues where many similar

692 cell subtypes exist, strong collinearity among the subtypes can render the model sensitive to

693 noise and prone to overfitting. AdRoit handles this problem by including an L2 norm of the

694 mixing proportions as the regularization component in the loss function. For a compound

695 transcriptome sample $j$, the loss function is given by,

696

$$
L_{j}\left(\beta_{1}, \ldots, \beta_{K} \mid y_{i j}, w_{i}^{C}, w_{i}^{S}, r_{i}, \widehat{\mu_{l k}}\right)=\sum_{i}^{I} w_{i}^{C} \cdot w_{i}^{S} \cdot\left(y_{i j}-r_{i} \cdot \sum_{k}^{K} \beta_{k} \widehat{\mu_{l k}}\right)^{2}+\sum_{k}^{K} \beta_{k}^{2}
$$

697 where $i$ is the index of a gene among the selected genes used for the deconvolution; $y_{i j}, \beta_{k}, r_{i}$,

698 and $\widehat{\mu_{l k}}$ are the same as defined in eq. $14 ; w_{i}^{S}$ and $w_{i}^{C}$ are computed as described above in eq. 7

699 and 11, respectively. The $\beta_{k}$ coefficients are estimated by minimizing the loss function subject

700 to the constraint $\beta_{1}, \ldots, \beta_{K}>0$,

701

$$
\widehat{\beta_{1}}, \ldots, \widehat{\beta_{K}}=\underset{\beta_{1}, \ldots, \beta_{K}}{\operatorname{argmax}} \beta_{\beta_{1}, \ldots, \beta_{K}>0} L
$$

702 The minimization is done by a gradient projection method proposed by Byrd et al ${ }^{60}$. We derive

703 the gradient function by taking the partial derivative of the loss function with respect to $\beta_{k}$,

704

$$
G_{k}=\nabla_{\beta_{k}} L_{j}=-2 \sum_{i}^{I} r_{i} \cdot \widehat{\mu_{l k}} \cdot w_{i}^{C} \cdot w_{i}^{S} \cdot\left(y_{i j}-r_{i} \cdot \sum_{k}^{K} \beta_{k} \widehat{\mu_{l k}}\right)+2 \beta_{k}
$$

AdRoit uses the function 'optim' from the R package 'stats' to perform the minimization ${ }^{61}$, 


$$
\theta_{k}=\frac{\widehat{\beta_{k}}}{\sum_{k}^{K} \widehat{\beta_{k}}}
$$

709 Each mixture RNA-Seq sample $j$ is independently estimated by the model described above.

711 Evaluation statistics

712 We compared the estimated cell type proportions with the ground truth by calculating the

713 following four statistics. The mean absolute difference (mAD) and root mean square deviation

714 (RMSD) are given by,

where $\theta_{k}$ and $\theta_{k}^{0}$ are the estimated proportions and true proportions, respectively.

Pearson correlation coefficient is computed as,

$$
\rho_{p}=\frac{\sum_{k}^{K}\left(\theta_{k}-\overline{\theta_{k}}\right)\left(\theta_{k}^{0}-\overline{\theta_{k}^{0}}\right)}{\sqrt{\sum_{k}^{K}\left(\theta_{k}-\overline{\theta_{k}}\right)^{2}} \sqrt{\sum_{k}^{K}\left(\theta_{k}^{0}-\overline{\theta_{k}^{0}}\right)^{2}}}
$$

respectively. Spearman correlation coefficient is given by,

$$
\rho_{s}=\frac{\sum_{k}^{K}\left(r_{k}-\overline{r_{k}}\right)\left(r_{k}^{0}-\overline{r_{k}^{0}}\right)}{\sqrt{\sum_{k}^{K}\left(r_{k}-\overline{r_{k}}\right)^{2}} \sqrt{\sum_{k}^{K}\left(r_{k}^{0}-\overline{r_{k}^{0}}\right)^{2}}}
$$

723 where $r_{k}$ is the rank of $\theta_{k}$. 
726 Bulk RNA-Seq data used for the benchmarking were synthesized by adding up the raw UMI

727 counts per gene from all cells within a subject. Let $t_{k}$ denote a cell of cell type $k, t_{k} \in 1, \ldots, T_{k}$,

728 where $T_{k}$ is the total number of cells in cell type $k$. Let $Y_{i j}^{B}$ be the count of gene $i$ in a

729 synthesized bulk sample associated with the subject $j$, and $X_{i j t_{k}}$ be the UMI count of gene $i$ in

730 cell $t_{k}$ within the subject $j$, then

731

$$
Y_{i j}^{B}=\sum_{k}^{K} \sum_{t_{k}}^{T_{k}} X_{i j t_{k}}
$$

732 The true proportion of cell type $k$ is given by,

733

$$
\theta_{k}^{0}=\frac{T_{k}}{\sum_{k}^{K} T_{k}}
$$

735 To simulate the spatial transcriptomic spots, we first sampled 10 cells without replacement

736 from each cell type, then weighed them by their respective mixing proportions before adding

737 them up. For example, to simulate a spot with $p_{k}$ percent of cell type $k$, the count $Y_{i j}^{S}$ of gene $i$

738 in a spatial spot $j$ is given by,

$$
Y_{i j}^{S}=\sum_{k}^{K} p_{k} \sum_{n=1}^{10} X_{i k n}
$$

740 where $X_{i k n}$ is the UMI count of gene $i$ in a selected cell $n$ of cell type $k$. For each mixing scheme,

741 the simulation was repeated 100 times.

\section{Single cell RNA sequencing of mouse dorsal root ganglion}

745 As described previously ${ }^{62}$, lumbar DRGs were isolated from five 18-week old adult C57BL/6

746 mice (three males and two females) and transferred to a dissociation buffer (Dulbecco's 
747 modified Eagle's medium supplemented with 10\% heat-inactivated Fetal Calf Serum) (Gibco; cat

748 \# A38400-02). To generate a single cell suspension, DRGs were subjected to a 2 step-enzymatic

749 dissociation followed by a mechanical dissociation. In brief, DRGs were first incubated with

$750 \quad 0.125 \%$ collagenase P from Clostridium histolyticum (Roche Applied Science; cat \#

751 11249002001) for 90 minutes in an Eppendorf Thermomixer C $\left(37^{\circ} \mathrm{C}\right.$; intermittent $750 \mathrm{rpm}$

752 shaking for about 10 sec every 2 minutes). Then, DRGs were transferred to a Hank's Balanced

753 Salt Solution (HBSS, $\mathrm{Mg}^{2+}$ and $\mathrm{Ca}^{2+}$ free; Invitrogen) supplemented with $0.25 \%$ Trypsin

754 (Worthington biochemical corp.; cat \# LSo03707) and 0.0025\% EDTA and incubated for 10

755 minutes at $37^{\circ} \mathrm{C}$ in the Eppendorf Thermomixer C. Trypsin was neutralized by the addition of 2.5

$756 \mathrm{mg} / \mathrm{ml} \mathrm{MgSO}$ (Sigma; cat \#M-3937) and DRGs were triturated with Pasteur pipettes. The

757 resulting cell suspension was passed through a $70 \mu \mathrm{m}$ mesh filter to remove remaining chunks

758 of tissues and centrifuged for 5 minutes at $2500 \mathrm{rpm}$ at room temperature. The pellet was

759 resuspended in HBSS ( $\mathrm{Ca}^{2+}, \mathrm{Mg}^{2+}$ free; Invitrogen) and the cell suspension was run on a $30 \%$

760 Percoll Plus gradient (Sigma GE17-5445-02) to further remove debris. Finally, cells were

761 resuspended in PBS supplemented with $0.04 \%$ BSA at a concentration of 200 cells/ $\mu$ l and cell

762 viability was determined using the automated cell analyzer NucleoCounter ${ }^{\circledR}$ NC-250 ${ }^{\text {TM }}$. The

763 suspended single cells were loaded on a Chromium Single Cell Instrument (10X Genomics) with

764 about 6000 cells per lane to minimize the presence of doublets. $2000-3000$ cells per lane were

765 recovered. RNA-Seq libraries were constructed using Chromium Single Cell 3' Library, Gel Beads

766 \& Multiplex Kit (10X Genomics). Single end sequencing was performed on Illumina NextSeq500.

767 Read 1 starts with a 26-bp UMI and cell barcode, followed by an 8-bp i7 sample index. Read 2

768 contains a 55-bp transcript read. Sample de-multiplexing, alignment, filtering, and UMI 
769

770

771

772

773

774

775

776

777

778

779

780

781

782

783

784

785

786

787

788

789

790

counting were conducted using Cell Ranger Single-Cell Software Suite ${ }^{63}$ (10X Genomics, v2.0.0). Mouse mm10 Genome assembly and UCSC gene model were used for the alignment.

\section{Data preprocessing}

DRG single cell data

The UMI data output from the Cell Ranger Single-Cell Software Suite (10X Genomics, v2.0.0) was analyzed using Seurat package ${ }^{64}$ to assess the cell quality and identify cell types, similar to what was described previously ${ }^{42}$. Cells with the number of detected genes less than 500 or over 15000, or with a UMI ratio of mitochondria encoded genes versus all genes over 0.1 were also removed. The UMI data was normalized by the 'NormalizeData' method in Seurat with default settings. To avoid potential sample-to-sample variation caused by the technical variation at various experiment steps, we employed Seurat data integration method. The top 2000 variable genes of each of the 5 DRG samples were identified using 'FindVariableFeatures' with selection. method='vst'. Based on the union of these variable genes, the anchor cells in each sample were identified by 'FindIntegrationAnchors'. All the samples were then integrated by 'IntegrateData'. We subsequently scaled the integrated data ('ScaleData') and performed dimension reduction ('RunPCA'). Cells were then clustered based on the first 15 principal components by applying 'FindNeighbors' and 'FindClusters' (resolution=0.6, algorithm=1). Marker genes for each cluster were identified using 'FindAllMarkers'. Parameters were used such that these genes were expressed in at least $25 \%$ of the cells in the cluster, and on average 2 -fold higher than the rest of cells with a multiple-testing adjusted Wilcoxon test $p$ value of less than 0.01. The specificity of the canonical cell type-specific genes or cell cluster-specific genes 
791 were further examined by visualizations (Supplementary Fig. 4) and used to define the cell type

792 for each cluster. At the end, the original UMI data from 17271 genes and 3352 cells that passed

793 the quality control were organized into a matrix (genes as rows and cell identifiers as columns).

794 This matrix, together with the cell type label for each cell therein, were loaded into AdRoit as

795 the reference profiles.

797 Mouse brain single cell atlas data

798 The scRNA-Seq reference data of the mouse brain were obtained from Zeisel et. al ${ }^{32}$. Among all

799 the available data, we only retained 96,572 cells that were acquired from the brain regions, had

800 an assigned cell type by the authors and a minimal total UMI of 1000 . These cells corresponded

801 to 183 clusters at the finest taxonomy level in the original study. As many of the clusters are

802 highly similar, we decided to merge some of them to simplify the reference landscape. First, the

803 top 50 cluster enriched markers were derived using Scanpy ${ }^{65}$ via the 'rank_genes_groups'

804 function (method='wilcoxon'), following the normalization ('normalize_per_cell'), log

805 transformation ('log $1 p^{\prime}$ ) and regressing out ('regress_out') the variances associated with the

806 total UMI and the percentage of mitochondrial chromosome encoded genes per cell. Then, the

807 pair-wise overlapping $\mathrm{p}$-values among the clusters were calculated using the top 50 marker

808 genes assuming a hypergeometric null distribution. Last, clusters with overlapping p-values

809 more significant than 1e-10 were merged and new names were assigned by combinedly

810 considering the original annotation, the molecular features and the specificity to certain brain

811 regions. A total of 46 cell types were determined that cover all the 12 brain regions and their

812 important substructures ${ }^{40}$ (Supplementary Table 7). To make the reference dataset more 
813 manageable in size and more balanced in the representation of cell types, we down sampled

814 each cluster to no more than 360 cells. A final set of 14,666 cells over 46 cell types was used for

815 the deconvolution of the mouse brain spatial transcriptome data.

816

817 Human Islets single cell and bulk RNA-Seq data

818 We used 1492 high quality human islets single cells and the associated annotations from Xin et

$819 \mathrm{al}^{41}$. The RPKM expression table was directly downloaded and used as-is. The RNA-FISH data

820 was also from this study ${ }^{41}$. The real bulk RNA-Seq data of human islets was acquired from a

821 large scale study referred in multiple publications ${ }^{41,43,44}$. We only included the data from donors

822 with a valid $\mathrm{HbA} 1 \mathrm{C}$ level measurement in the regression analysis of the Beta cell proportion

823 with respect to the HbA1C level (Fig. 5c, Supplementary Table 13).

824

825 Trabecular Meshwork single cell data

826 We acquired the UMI counts, tSNE coordinates and the cell type annotation of 8759 high

827 quality cells from the authors of Patel et al ${ }^{42}$. Details of the quality control and the cell type

828 identification were presented in the original publication.

829

830

Mouse Brain Spatial transcriptomics data by 10x Visium platform

831 The filtered count matrix, tissue image and the spatial coordinates of a coronal section of an

832 adult C57BL/6 mouse brain were download from the 10x Genomics website (see Data

833 availability). A total of 2698 in-tissue spots were provided in the data set and used as-is. 
835

836

837

838

839

840

841

842

843

844

845

846

847

848

849

850

851 (https://support.10xgenomics.com/spatial-gene-

852

853

854 Code availability

855 AdRoit's source code is available on Github (https://github.com/TaoYang-dev/AdRoit).

The ISH images were directly downloaded from Allen mouse Brain Atlas ${ }^{40}$ by searching the gene names. The images were used without further editing except for cropping.

\section{Data availability}

The mouse DRG single cell data were deposited at NCBI GEO (accession number: GSE163252).

The bulk RNA-Seq and RNA-FISH data for human pancreatic islets were initially published as aggregated data where the data processing and experimental procedure were described

therein ${ }^{41,43,44}$. We acquired the individual sample data from the authors and released them along with the current study (Supplementary Table 10 and Supplementary Table 12). The other public data analyzed in this study were obtained using GEO accession number GSE81608 (human pancreatic islets single cell data), NCBI SRA accession number PRJNA616025 (human trabecular meshwork single cell data), and NCBI SRA accession number SRP135960 (mouse brain single cell data). The 10x Genomics PBMC data was downloaded from the link (https://support.10xgenomics.com/single-cell-gene-expression/datasets/1.1.0/pbmc3k), and the Visium mouse brain spatial transcriptomic data was downloaded from the link expression/datasets/1.1.0/V1_Adult_Mouse_Brain_Coronal_Section). 
857

858 The statistical analyses were done with R statistical software (v3.6.0 $)^{61}$ and python (v3.7.2) ${ }^{66}$.

859 The packages used include Seurat $(v 3.0 .1)^{64}$, scanpy $(v 1.6 .0)^{65}$, dplyr (v0.8.0.1 $)^{67}$, doParallel

860 (v1.0.14) $)^{68}$, data.table $(v 1.12 .4)^{69}$, fitdistrplus $(v 1.1-1)^{58}$, nnls $(v 1.4)^{59}$, MuSiC (v0.1.1 $)^{22}$

861 BisqueRNA (v1.0.4) ${ }^{21}$, SPOTlight $(v 1.0 .4)^{38}$, Cell2location (v0.05-alpha) $)^{39}$, Stereoscope $\left(v \_03\right)^{23}$.

\section{Reference}

864 1. Wang, Z., Gerstein, M. \& Snyder, M. RNA-Seq: A revolutionary tool for transcriptomics.

$865 \quad$ Nature Reviews Genetics (2009) doi:10.1038/nrg2484.

866 2. Chu, G. C., Kimmelman, A. C., Hezel, A. F. \& DePinho, R. A. Stromal biology of pancreatic

867 cancer. Journal of Cellular Biochemistry (2007) doi:10.1002/jcb.21209.

868 3. Bussard, K. M., Mutkus, L., Stumpf, K., Gomez-Manzano, C. \& Marini, F. C. Tumor-

869 associated stromal cells as key contributors to the tumor microenvironment. Breast

$870 \quad$ Cancer Research (2016) doi:10.1186/s13058-016-0740-2.

871 4. Munn, D. H. \& Bronte, V. Immune suppressive mechanisms in the tumor

872 microenvironment. Current Opinion in Immunology (2016)

873 doi:10.1016/j.coi.2015.10.009.

874 5. Gonzalez, H., Hagerling, C. \& Werb, Z. Roles of the immune system in cancer: From tumor

875 initiation to metastatic progression. Genes and Development (2018)

876 doi:10.1101/GAD.314617.118.

877 6. Garner, H. \& de Visser, K. E. Immune crosstalk in cancer progression and metastatic 878 spread: a complex conversation. Nature Reviews Immunology (2020) 
doi:10.1038/s41577-019-0271-z.

880 7. Singh, U. P. et al. Chemokine and cytokine levels in inflammatory bowel disease patients.

$881 \quad$ Cytokine (2016) doi:10.1016/j.cyto.2015.10.008.

882 8. Van Lint, P. \& Libert, C. Chemokine and cytokine processing by matrix metalloproteinases and its effect on leukocyte migration and inflammation. J. Leukoc. Biol. (2007) doi:10.1189/jlb.0607338.

885 9. Zelová, H. \& Hošek, J. TNF- $\alpha$ signalling and inflammation: Interactions between old acquaintances. Inflammation Research (2013) doi:10.1007/s00011-013-0633-0.

10. Koelman, L., Pivovarova-Ramich, O., Pfeiffer, A. F. H., Grune, T. \& Aleksandrova, K. and phenotypic characterisation. Immun. Ageing (2019) doi:10.1186/s12979-019-0151-1.

11. Landskron, G., De La Fuente, M., Thuwajit, P., Thuwajit, C. \& Hermoso, M. A. Chronic inflammation and cytokines in the tumor microenvironment. Journal of Immunology Research (2014) doi:10.1155/2014/149185.

12. Ståhl, P. L. et al. Visualization and analysis of gene expression in tissue sections by spatial transcriptomics. Science (2016) doi:10.1126/science.aaf2403.

895 13. Vickovic, S. et al. High-definition spatial transcriptomics for in situ tissue profiling. Nat. Methods (2019) doi:10.1038/s41592-019-0548-y.

897 14. Tang, F. et al. mRNA-Seq whole-transcriptome analysis of a single cell. Nat. Methods (2009) doi:10.1038/nmeth.1315.

899 15. Denisenko, E. et al. Systematic assessment of tissue dissociation and storage biases in single-cell and single-nucleus RNA-Seq workflows. Genome Biol. (2020) 
doi:10.1186/s13059-020-02048-6.

902 16. Nguyen, Q. H., Pervolarakis, N., Nee, K. \& Kessenbrock, K. Experimental considerations

903 for single-cell RNA sequencing approaches. Frontiers in Cell and Developmental Biology

904 (2018) doi:10.3389/fcell.2018.00108.

905

17. Tanay, A. \& Regev, A. Scaling single-cell genomics from phenomenology to mechanism. Nature (2017) doi:10.1038/nature21350.

907

18. Abbas, A. R., Wolslegel, K., Seshasayee, D., Modrusan, Z. \& Clark, H. F. Deconvolution of blood microarray data identifies cellular activation patterns in systemic lupus erythematosus. PLoS One (2009) doi:10.1371/journal.pone.0006098.

19. Newman, A. M. et al. Robust enumeration of cell subsets from tissue expression profiles. Nat. Methods (2015) doi:10.1038/nmeth.3337.

912 20. Baron, M. et al. A Single-Cell Transcriptomic Map of the Human and Mouse Pancreas Reveals Inter- and Intra-cell Population Structure. Cell Syst. (2016) doi:10.1016/j.cels.2016.08.011.

915 21. Tsoucas, D. et al. Accurate estimation of cell-type composition from gene expression data. Nat. Commun. (2019) doi:10.1038/s41467-019-10802-z.

917 22. Wang, X., Park, J., Susztak, K., Zhang, N. R. \& Li, M. Bulk tissue cell type deconvolution with multi-subject single-cell expression reference. Nat. Commun. (2019) doi:10.1038/s41467-018-08023-x.

920 23. Andersson, A. et al. Single-cell and spatial transcriptomics enables probabilistic inference of cell type topography. Commun. Biol. 3, 565 (2020).

922 24. Newman, A. M. et al. Determining cell type abundance and expression from bulk tissues 
with digital cytometry. Nat. Biotechnol. (2019) doi:10.1038/s41587-019-0114-2.

924 25. Myung, I. J. Tutorial on maximum likelihood estimation. J. Math. Psychol. (2003)

925 doi:10.1016/S0022-2496(02)00028-7.

926 26. Bassett, R. \& Deride, J. Maximum a posteriori estimators as a limit of Bayes estimators. Math. Program. (2019) doi:10.1007/s10107-018-1241-0.

928 27. Zhao, Y. \& Simon, R. Gene expression deconvolution in clinical samples. Genome Medicine (2010) doi:10.1186/gm214.

930

28. Chiu, Y. J., Hsieh, Y. H. \& Huang, Y. H. Improved cell composition deconvolution method of bulk gene expression profiles to quantify subsets of immune cells. BMCMed.

932 Genomics (2019) doi:10.1186/s12920-019-0613-5.

933

29. Kang, K. et al. CDSeq: A novel complete deconvolution method for dissecting heterogeneous samples using gene expression data. PLoS Comput. Biol. (2019) doi:10.1371/journal.pcbi.1007510.

30. Qiao, W. et al. PERT: A Method for Expression Deconvolution of Human Blood Samples from Varied Microenvironmental and Developmental Conditions. PLoS Comput. Biol. (2012) doi:10.1371/journal.pcbi.1002838.

31. Zaitsev, K., Bambouskova, M., Swain, A. \& Artyomov, M. N. Complete deconvolution of cellular mixtures based on linearity of transcriptional signatures. Nat. Commun. (2019) doi:10.1038/s41467-019-09990-5. 
deconvolution of GTEx tissues powers discovery of disease and cell-type associated regulatory variants. Nat. Commun. (2020) doi:10.1038/s41467-020-14561-0.

947 34. Phipson, B., Zappia, L. \& Oshlack, A. Gene length and detection bias in single cell RNA sequencing protocols. F1000Research (2017) doi:10.12688/f1000research.11290.1.

35. Chen, G., Ning, B. \& Shi, T. Single-cell RNA-Seq technologies and related computational data analysis. Frontiers in Genetics (2019) doi:10.3389/fgene.2019.00317.

951 36. Chen, D. \& Plemmons, R. J. Nonnegativity constraints in numerical analysis. in The Birth of Numerical Analysis (2009). doi:10.1142/9789812836267_0008.

37. Jew, B. et al. Accurate estimation of cell composition in bulk expression through robust integration of single-cell information. Nat. Commun. (2020) doi:10.1038/s41467-02015816-6.

38. Elosua-Bayes, M., Nieto, P., Mereu, E., Gut, I. \& Heyn, H. SPOTlight: seeded NMF regression to deconvolute spatial transcriptomics spots with single-cell transcriptomes. Nucleic Acids Res. (2021) doi:10.1093/nar/gkab043.

39. Kleshchevnikov, V. et al. Comprehensive mapping of tissue cell architecture via integrated single cell and spatial transcriptomics. bioRxiv (2020).

961 40. Lein, E. S. et al. Genome-wide atlas of gene expression in the adult mouse brain. Nature (2007) doi:10.1038/nature05453.

963 41. Xin, Y. et al. RNA Sequencing of Single Human Islet Cells Reveals Type 2 Diabetes Genes. 964 Cell Metab. (2016) doi:10.1016/j.cmet.2016.08.018.

965 42. Patel, G. et al. Molecular taxonomy of human ocular outflow tissues defined by single966 cell transcriptomics. Proc. Natl. Acad. Sci. 117, 12856 LP - 12867 (2020). 
43. Xin, Y. et al. Pseudotime ordering of single human B-cells reveals states of insulin production and unfolded protein response. Diabetes (2018) doi:10.2337/db18-0365.

44. Gutierrez, G. D. et al. Gene signature of proliferating human pancreatic a cells. Endocrinology (2018) doi:10.1210/en.2018-00469.

971 45. Cerf, M. E. Beta cell dysfunction and insulin resistance. Frontiers in Endocrinology (2013) doi:10.3389/fendo.2013.00037.

46. Maedler, K. \& Donath, M. Y. Beta-cells in type 2 diabetes: a loss of function and mass. Hormone research (2004).

975 47. Donath, M. Y. et al. Mechanisms of $\beta$-cell death in type 2 diabetes. Diabetes (2005) doi:10.2337/diabetes.54.suppl_2.S108.

977 48. Calanna, S. et al. Alpha- and beta-cell abnormalities in haemoglobin A1c-defined prediabetes and type 2 diabetes. Acta Diabetol. (2014) doi:10.1007/s00592-014-0555-5.

49. Kanat, M. et al. The Relationship Between $\beta$-Cell Function and Glycated Hemoglobin. Diabetes Care 34, 1006 LP - 1010 (2011).

981 50. Nepton, S. Beta-Cell Function and Failure. in Type 1 Diabetes (2013). doi:10.5772/52153.

982 51. Dolenšek, J., Rupnik, M. S. \& Stožer, A. Structural similarities and differences between 983 the human and the mouse pancreas. Islets (2015) doi:10.1080/19382014.2015.1024405.

984 52. Lein, E. S. et al. Genome-wide atlas of gene expression in the adult mouse brain. Nature 985 445, 168-176 (2007).

986 53. Vieth, B., Parekh, S., Ziegenhain, C., Enard, W. \& Hellmann, I. A systematic evaluation of single cell RNA-Seq analysis pipelines. Nat. Commun. (2019) doi:10.1038/s41467-01912266-7. 
989

990

991

992

993

994

995

996

997

998

999

1000

1001

1002

1003

1004

1005

1006

1007

1008

1009

1010

54. Wang, J., Roeder, K. \& Devlin, B. Bayesian estimation of cell type-specific gene expression with prior derived from single-cell data. Genome Res. (2021) doi:10.1101/gr.268722.120.

55. Anders, S. \& Huber, W. Differential expression analysis for sequence count data. Genome Biol. (2010) doi:10.1186/gb-2010-11-10-r106.

56. Hafemeister, C. \& Satija, R. Normalization and variance stabilization of single-cell RNASeq data using regularized negative binomial regression. Genome Biol. (2019) doi:10.1186/s13059-019-1874-1.

57. Svensson, V. Droplet scRNA-Seq is not zero-inflated. Nature Biotechnology (2020) doi:10.1038/s41587-019-0379-5.

58. Delignette-Muller, M. L. \& Dutang, C. fitdistrplus: An R package for fitting distributions. J. Stat. Softw. (2015) doi:10.18637/jss.v064.i04.

59. Mullen, Katharine M., I. H. M. van S. nnls: The Lawson-Hanson algorithm for nonnegative least squares (NNLS). R Packag. version 1.4 (2012).

60. Byrd, R. H., Lu, P., Nocedal, J. \& Zhu, C. A Limited Memory Algorithm for Bound Constrained Optimization. SIAM J. Sci. Comput. (1995) doi:10.1137/0916069.

61. The R Core Team. R: A Language and Environment for Statistical Computing. $R$ Foundation for Statistical Computing (2019).

62. Alessandri-Haber, N. et al. Hypotonicity induces TRPV4-mediated nociception in rat. Neuron (2003) doi:10.1016/S0896-6273(03)00462-8.

63. Zheng, G. X. Y. et al. Massively parallel digital transcriptional profiling of single cells. Nat. Commun. (2017) doi:10.1038/ncomms14049.

64. Stuart, T. et al. Comprehensive Integration of Single-Cell Data. Cell (2019) 
doi:10.1016/j.cell.2019.05.031.

1012 65. Wolf, F. A., Angerer, P. \& Theis, F. J. SCANPY: Large-scale single-cell gene expression data analysis. Genome Biol. (2018) doi:10.1186/s13059-017-1382-0.

1014 66. van Rossum, G. \& Drake, F. L. Python 3 Reference Manual. Scotts Valley, CA (2009).

1015 67. Wickham, H. \& Francois, R. dplyr: A Grammar of Data Manipulation. R Packag. version 0.4.2. (2015).

68. Weston, S., Calaway, R. \& Tenenbaum, D. doParallel: Foreach Parallel Adaptor for the Parallel Package. Cran (2014).

69. Dowle, M. \& Srinivasan, A. data.table: Extension of 'data.frame'. R Package Version 1.12.8. Manual (2019).

\section{Acknowledgements}

1023 We thank Yurong Xin for pointing us to the relevant public data resource. We also thank Gabor 1024 Halasz and Yuan Zhu for the helpful discussions regarding the memory usage, and Shawn 1025 Mishra for the manuscript proofreading.

\section{Author contributions}

1028 Y.B., T.Y., N.A.-H., M.L.-F., L.E.M. and G.S.A. designed the research. T.Y., Y.B., and W.F.

1029 developed the algorithm. T.Y., Y.B. and J.K. participated in the data analysis. M.S. and R.B.

1030 performed the DRG tissue collection. C.A. performed the single cell library preparation and 1031 sequencing experiment. Y.B., T.Y. and N.A.-H. wrote the manuscript. 


\section{Competing interests}

1034 T.Y., Y.B., W.F. and G.S.A. have filed a patent application relating to the AdRoit computational

1035 framework. M.L.-F. is an employee of Cellular Longevity. All other authors are employees

1036 and/or shareholders of Regeneron Pharmaceuticals, although the manuscript's subject matter

1037 does not have any relationship to any products or services of this corporation.

1038

1039 Figure legends

1040 Fig. 1: Schematic representation of AdRoit computational framework. a, AdRoit inputs

1041 compound (bulk or spatial) RNA-Seq data, single cell RNA-Seq data and cell type annotations. It

1042 first selects informative genes and estimates their means and dispersions, then computes the

1043 cell type specificity of genes. Depending on the availability of multiple samples, cross-sample

1044 gene variability is derived from either the compound RNA-Seq, or the single cell data (dashed

1045 arrow; see also Methods). Lastly the gene-wise scaling factors are computed to correct the

1046 platform bias between the compound and the single cell RNA-Seq data. These quantities are

1047 used in a weighted regularized model to infer the cell type composition. $\mathbf{b}, \mathrm{A}$ mock example to

1048 illustrate the role of the gene-wise scaling factor. Ideally, an accurate estimation of the cell

1049 proportions should be represented by the slope of the green line, however fitting in the

1050 presence of outlier genes would result in the red line. Outlier genes exist because the platform

1051 bias affects genes differently. AdRoit adopts an adaptive learning approach that first learns a

1052 coarse estimation of the slope (red line), from which the gene-wise scaling factors are derived

1053 and applied to the outlier genes, moving them toward the green line. The more deviated the 
1054 gene, the larger the correction (i.e., longer arrows). After the adjustment, the new estimated

1055 slope (blue line) is closer to the truth (green line), thus is a more accurate estimation.

Fig.2: Benchmark on simulated bulk data generated from the trabecular meshwork (TM)

AdRoit has the smallest differences from the true cell type proportion and the smallest variance

1061 of estimates across the 8 donors. For each cell type, a dot on the graph denotes a donor, and

1062 the bars represent the $1.5 \times$ interquartile ranges. The reference used for deconvoluting each

1063 synthetic bulk sample excludes the cells used to synthesize that sample (leave-one-out).

Fig. 3: AdRoit can achieve a high granularity and exhibits high sensitivity and specificity in

complex tissues. a. AdRoit is accurate in deconvoluting the simulated bulk samples that contain

1067 a mixture of similar cell types from myeloid or lymphoid lineage. The vertical dashed lines

1068 indicate the true mixing proportions. CD14 ${ }^{+}$monocytes, FCGR3A ${ }^{+}$monocytes and dendritic cells

1069 (DC) were mixed under three schemes of proportions: 0.33:0.33:0.33 ( $\mathrm{mix} 0)$, 0.1:0.45:0.45

1070 (mix1) and 0.1:0.3:0.6 (mix2). The same ratios were applied to the mixtures of naïve $\mathrm{CD}^{+} \mathrm{T}$,

1071 memory $\mathrm{CD}^{+} \mathrm{T}$ and $\mathrm{CD} 8^{+}$cells. $\mathbf{b}$, AdRoit's estimates are more accurate and specific than those

1072 from Bisque, MuSiC and SPOTlight on synthetic samples that contain only 6 out of the 12 cell

1073 types. The deconvolution was done using all 12 cell types as the reference. A pair of size-

1074 matched blue (true value) and red (estimated value) bubbles indicate an accurate prediction.

1075 Red-only and blue-only bubbles mark false positives and false negatives, respectively. d, 
1076 Receiver operating characteristic (ROC) curve shows AdRoit has a significantly higher area

1077 under the curve (AUC) than other methods, meaning better sensitivity and specificity. e,

1078 Scatterplots between the ground truth and the deconvoluted cell proportions in the simulated

1079 bulk samples of high complexity (mixtures of 30 cell types). f, ROC curves show AdRoit has the

1080 best AUC among all methods on highly complex cell constitutions.

1081

\section{Fig. 4: Benchmark on scRNA-Seq data from dorsal root ganglion (DRG) where many closely}

1083 related subtypes of neuronal cells exist. a, 14 cell types are identified from scRNA-Seq samples

1084 of 5 mice, including multiple subtypes of neurofilaments (NF), peptidergic (PEP), and non-

1085 peptidergic (NP) neurons. $\mathbf{b}$, Benchmarking with the synthetic data shows the cell type

1086 proportions inferred by AdRoit are highly accurate. In particular, AdRoit remains a high

1087 accuracy when the cells are rare (e.g., < 5\%; see also the zoom-in inserts). Each dot represents a

1088 cell type from one sample. c, For each sample, mAD, RMSD, Pearson and Spearman correlations

1089 are compared across four methods. AdRoit has the lowest mAD and RMSD, and the highest

1090 Pearson and Spearman correlations. In addition, AdRoit's estimation is the most stable across

1091 samples. Each dot on the boxplot is a sample. Same samples are chained by the dotted lines

1092 across the metrics. The deconvolution was done by using the leave-one-out strategy.

1093

1094 Fig. 5: AdRoit is more accurate and sensitive on spatial spots simulated from real DRG cells. a,

1095 Estimations from AdRoit, Cell2location,Stereoscope and SPOTlight on simulated spatial spots

1096 that contain 5 PEP neuron subtypes. True mixing proportions are denoted by the red dashed

1097 lines. Three schemes are presented: 1) the proportions of 5 PEP cell types are the same and 
1098

1099

1100

1101

1102

1103

1104

1105

1106

1107

1108

1109

1110

1111

1112

1113

1114

1115

1116

1117

1118

1119

equal to 0.2; 2) PEP1_Dcn is 0.1 and the other 4 are 0.225 ; 3) PEP1_Dcn and

PEP1_S100a11.Tagln2 are 0.1, PEP1_Slc7a3.Sstr2 and PEP2_Htr3a.Sema5a 0.2 are 0.2, and

PEP3_Trpm8 is 0.4. b, The performance of AdRoit, Cell2location, Stereoscope, and SPOTlight in estimating rare cell populations in the spatial spots. The spots contain a mixture of three PEP

cell subtypes (i.e., PEP1_Slc7a3.Sstr2, PEP2_Htr3a.Sema5a and PEP3_Trpm8), with the percent of PEP3_Trpm8 ranging from $1 \%$ to $10 \%$ and the other two cell types sharing the remaining proportion equally. c, Compare the rate of detecting rare cells in simulated spots. An inferred percent greater than $0.5 \%$ is deemed as a positive detection. Six sets of cell mixtures are employed: NF_Calb1 with NF_Pvalb and NF2_Ntrk2.Necab2 (NP subtypes), NP_Mrgpra3 with NP_Mrgprd and NP_Nts (NP subtypes), PEP3_Trpm8 with PEP1_Slc7a3.Sstr2 and PEP2_Htr3a.Sema5a (PEP subtypes), NF_Calb1 with Th, satellite glia and endothelial (NF_Calb1 + others), NP_Mrgpra3 with Th, satellite glia and endothelial (NP_Mrgpra3 + others), and PEP_Trpm8 with Th, satellite glia and endothelial (PEP_Trpm8 + others). In each set, the first cell type listed is the target of detection and varies its percent from $1 \%$ to $10 \%$. The rest cell types split the remaining proportion evenly. The red dashed lines mark the detection rate of $90 \%$.

\section{Fig. 6: Applications to real-world bulk RNA-Seq data and mouse brain spatial transcriptome}

data. a, The deconvoluted cell compositions in the real bulk RNA-Seq data of human Islets are highly reproducible for the repeated samples from the same donor. $\mathbf{b}$, AdRoit estimation of the cell type proportions agrees with the RNA-FISH measurements. c, AdRoit-inferred Beta cell proportions in type 2 diabetes patients are significantly lower than those in healthy subjects. In 
1120 addition, the estimated proportions have a significant negative linear association with the

1121 donor HbA1C levels. d, The spatial mapping of four mouse brain cell types is consistent with the

1122 locations of four region-specific markers shown on the ISH images obtained from Allen mouse

1123 brain atlas $^{40}$. The four genes, Spink8, C1ql2, Clic6 and Synpo2, were identified by Zeisel et al ${ }^{32}$ as

1124 markers of the hippocampal field CA1, dentate gyrus, choroid plexus and thalamus,

1125 respectively.

1126

1127

1128

1129

1130

1131

1132

1133 